\title{
Inclusion of quasi-vertex views in a brain-dedicated multi-pinhole SPECT system for improved imaging performance
}

To cite this article before publication: Benjamin Auer et al 2020 Phys. Med. Biol. in press https://doi.org/10.1088/1361-6560/abc22e

\section{Manuscript version: Accepted Manuscript}

Accepted Manuscript is "the version of the article accepted for publication including all changes made as a result of the peer review process, and which may also include the addition to the article by IOP Publishing of a header, an article ID, a cover sheet and/or an 'Accepted

Manuscript' watermark, but excluding any other editing, typesetting or other changes made by IOP Publishing and/or its licensors"

This Accepted Manuscript is $\odot 2020$ Institute of Physics and Engineering in Medicine.

During the embargo period (the 12 month period from the publication of the Version of Record of this article), the Accepted Manuscript is fully protected by copyright and cannot be reused or reposted elsewhere.

As the Version of Record of this article is going to be / has been published on a subscription basis, this Accepted Manuscript is available for reuse under a CC BY-NC-ND 3.0 licence after the 12 month embargo period.

After the embargo period, everyone is permitted to use copy and redistribute this article for non-commercial purposes only, provided that they adhere to all the terms of the licence https://creativecommons.org/licences/by-nc-nd/3.0

Although reasonable endeavours have been taken to obtain all necessary permissions from third parties to include their copyrighted content within this article, their full citation and copyright line may not be present in this Accepted Manuscript version. Before using any content from this article, please refer to the Version of Record on IOPscience once published for full citation and copyright details, as permissions will likely be required. All third party content is fully copyright protected, unless specifically stated otherwise in the figure caption in the Version of Record.

View the article online for updates and enhancements. 


\title{
Inclusion of Quasi-Vertex Views in a Brain-Dedicated Multi-Pinhole SPECT System for Improved Imaging Performance
}

\author{
Benjamin Auer ${ }^{1}$, Navid Zeraatkar ${ }^{1}$, Justin C. Goding ${ }^{1}$, Arda Könik ${ }^{2}$, Timothy \\ J. Fromme ${ }^{3}$, Kesava S. Kalluri ${ }^{1}$, Lars R. Furenlid ${ }^{4,5}$, Phillip H. Kuo ${ }^{5}$, and \\ Michael A. King ${ }^{1}$ \\ ${ }^{1}$ Department of Radiology, University of Massachusetts Medical School, Worcester, MA, USA, 01655. \\ ${ }^{2}$ Department of Imaging, Dana Farber Cancer Institute, Boston, MA, USA, 02215. ${ }^{3}$ Worcester Polytechnic \\ Institute, Worcester, MA, USA, 01609. ${ }^{4}$ Wyant College of Optical Sciences, University of Arizona, Tucson, \\ AZ, USA, 85721. ${ }^{5}$ Department of Medical Imaging, University of Arizona, Tucson, AZ, USA, 85724.
}

E-mail: Ben.Auer@umassmed.edu

July 2020

\begin{abstract}
With brain-dedicated multi-detector systems employing pinhole apertures the usage of detectors facing the top of the patient's head (i.e., quasi-vertex/views) can provide the advantage of additional viewing from close to the brain for improved detector coverage. In this paper, we report the results of simulation and reconstruction studies to investigate the impact of the quasi-vertex views on the imaging performance of AdaptiSPECT-C, a brain-dedicated stationary SPECT system under development. In this design, both primary and scatter photons from regions located inferior to the brain can contribute to SPECT projections acquired by the quasi-vertex views, and thus degrade AdaptiSPECT-C imaging performance. In this work, we determined the proportion, origin, and nature (i.e., primary, scatter, and multiple-scatter) of counts emitted from structures within the head and throughout the body contributing to projections from the different AdaptiSPECT-C detector rings, as well as from a true vertex view detector. We simulated phantoms used to assess different aspects of image quality (i.e., uniform activity concentration sphere, and Derenzo), as well as anthropomorphic phantoms with different count levels emulating clinical ${ }^{123}$ I activity distributions (i.e., DaTscan and perfusion). We determined that attenuation and scatter in the patient's body greatly diminish the probability of the photons emitted outside the volume of interest reaching to detectors and being recorded within the $15 \%$ photopeak energy window. In addition, we demonstrated that the inclusion of the residual of such counts in the system acquisition does not degrade visual interpretation or quantitative analysis. The addition of the quasi-vertex detectors increases volumetric sensitivity, angular sampling, and spatial resolution leading to significant enhancement in image quality, especially in the striato-thalamic and superior regions of the brain. Besides, the use of quasi-vertex detectors improves the recovery of clinically relevant metrics such as the striatal binding ratio and mean activity in selected cerebral structures. Our findings proving the usefulness of the quasi-vertex ring for brain imaging with ${ }^{123} \mathrm{I}$ agents can be generalized to other commonly used SPECT imaging agents labelled with isotopes, such as ${ }^{99 \mathrm{~m}} \mathrm{Tc}$ and likely ${ }^{111} \mathrm{I}$.

Keywords: Brain SPECT, ${ }^{123}$ I-labeled tracers, AdaptiSPECT-C, vertex and quasi-vertex view, multipinhole, image quality assessment
\end{abstract}

\section{Introduction}

The use of SPECT in brain imaging has proven to be an indispensable tool for diagnosis and evaluation of disease progression, treatment monitoring, and follow-up of patients with brain disorders (Catafau 2001, Camargo 2001, Goorden et al. 2009), such as Parkinsonism (Bajaj et al. 2013, Suwijn et al. 2015), Huntington chorea (Montoya et al. 2006), epilepsy (Van Paesschen et al. 2007), dementias (Petrella et al. 2003, Yeo et al. 2013), cerebrovascular disease (Moretti et al. 1994), and traumatic brain injury (Kant et al. 1997). Presently, the two main clinical applications of brain SPECT are cerebral blood perfusion assessment and dopaminergic neuro-receptor uptake measurement with DaTscan (Goorden et al. 2009, Gonzalez et al. 2018). DaTscan ( ${ }^{123}$ I-Ioflupane; Medi-Physics Inc. dba GE Healthcare, Arlington Heights, IL, USA) imaging is of interest in the clinical differential diagnosis of Parkinsonism (Bajaj et al. 2013, Kuribara et al. 2020), Alzheimer's disease (Djang et al. 2012), and dementia with Lewy bodies (Pimlott \& Sutherland 2011). Perfusion imaging with ${ }^{123} \mathrm{I}$-IMP, ${ }^{99 \mathrm{~m}} \mathrm{Tc}-\mathrm{HMPAO}$ or ${ }^{99 \mathrm{~m}} \mathrm{Tc}-\mathrm{ECD}$ agents is routinely used for clinical 
evaluation of cerebrovascular disease, dementia, epilepsy, head injury, and in the differential diagnosis of Alzheimer's disease (Catafau 2001, Petrella et al. 2003, Khalil et al. 2011). For example, postictal subtraction SPECT is effective for localizing seizure focus for operative planning in epilepsy (Fukuma et al. 2020). The use of perfusion and neuro-receptor imaging in psychiatry has shown considerable interest in the evaluation of obsessive-compulsive disorder, Gilles de la Tourette's syndrome, schizophrenia, depression, panic disorder, anxiety, or attention deficit hyperactivity disorder (Bonne et al. 1992, Camargo 2001, Amen et al. 2012, Santra \& Kumar 2014).

Most brain SPECT imaging is performed using general-purpose dual-headed scanners equipped with low-energy-high-resolution parallel-hole collimators (LEHR) (Goorden et al. 2009, Khalil et al, 2011). These systems provide a poor spatial resolution and sensitivity trade-off due to the use of absorptive collimation, and limited field of view due to high brain reach $(\sim 7-8 \mathrm{~cm})$ (Goorden et al. 2009, Khalil et al. 2011). Given the small size of brain structures a significant enhancement in sensitivity and spatial resolution would improve lesion detection and quantification, early diagnosis, treatment monitoring and reduction in equivocal reports (Warwick 2004, Goorden et al. 2009, Suwijn et al. 2015, Chen et al. 2017). Achieving higher sensitivity could also be of interest to reduce the injected dose and/or imaging time, leading to improved cost-effectiveness while maintaining the same diagnostic impact (Farid et al. 2011).

In this context, the use of multi-pinhole collimation (MPC) has proven to be successful in significantly enhancing the clinical imaging of the brain, thyroid or heart (Beekman \& van der Have 2007, Van Audenhaege et al. 2015). MPC offers an excellent sensitivity to spatial resolution trade-off for brain imaging (Chen et al. 2019), while reducing the fraction of down-scatter counts (Könik, Auer, De Beenhouwer, Kalluri, Zeraatkar, Furenlid \& King 2019). Moreover, MPC provides improved penetration characteristics which makes its use more suited for high-energy photon-emitting isotope imaging (e.g., ${ }^{111} \mathrm{In},{ }^{123} \mathrm{I}$ ) (Van Audenhaege et al. 2013, Könik, Auer, De Beenhouwer, Kalluri, Zeraatkar, Furenlid \& King 2019). Research has focused on the development of high-resolution high-sensitivity multi-pinhole systems designed specifically for brain imaging, such as the CeraSPECT (Batis 2011, Stam et al. 2018, Adriaanse et al. 2018), the InspiraHD system (Stoddart \& Stoddart 1992, El Fakhri et al. 2006), and recently the G-SPECT (Beekman et al. 2015, Chen et al. 2018, Chen et al. 2019). Among these systems, the best performance was reported for the G-SPECT with a $3 \mathrm{~mm}$ spatial resolution associated to a $0.04 \%$ sensitivity in a central volume of the brain $(10 \mathrm{~cm}-$ diameter, $6 \mathrm{~cm}$-axial length cylindrical volume) (Chen et al. 2019). Multi-axis bed movement is required to ensure complete sampling of the whole-brain with this system.

Several research groups have proposed lower-cost alternatives based on adapting existing general-purpose systems for brain imaging by replacing one or more of their parallel-beam collimators by multi-pinhole collimators (Lee et al. 2014, Ogawa \& Ichimura 2014, King et al. 2016, Chen et al. 2017, Könik, Zeraatkar, Kalluri, Auer, Furenlid \& King 2019, Könik et al. 2018). However, due to overhead time introduced by mechanical detector-head rotation or imaging-bed translation, most of the systems listed above might not be suited for high-temporal-resolution dynamic imaging of the whole-brain and pharmacokinetic studies (Gullberg et al. 2010). Lastly, to the best of our knowledge only a small number of these systems presently available are capable of real-time adaptation of their configurations to a given task during clinical imaging, e.g., to optimize localization of tumours and/or perfusion defects (Li \& Meng 2011, Barrett et al. 2008).

We are constructing an innovative stationary dynamic system, AdaptiSPECT-C, specifically designed for multi-purpose brain imaging and capable of adapting in real-time the number of pinholes and the pinhole aperture radii for a given imaging task by using a shuttering mechanism. Since it images the entire brain without mechanical bed translation or camera rotation, we proposed the use of an additional MPC detector ring, referred to as the quasi-vertex ring $(\mathrm{QV})$, for significant sensitivity and sampling enhancement while maintaining high-resolution.

Historically, in planar scintigraphy the use of vertex views (i.e., imaging with the detector facing the top of the patient's head) was found to be useful for locating lesions in the brain (Overton et al. 1965, Holmes \& North 1969, Holmes 1971). However, while the advantages of incorporating the vertex view were noted, activity arising from organs and tissues inferior to the brain were problematic. For example, Ng et al., utilizing uniform Cramér-Rao lower bound analysis, determined an improvement in performance when projections from a vertex detector were combined in reconstruction with the standard transaxial views from a conventional SPECT system, especially for central locations ( $\mathrm{Ng}$ et al. 1996). However, they also pointed out the need to account for events in the vertex view arising from activity located in the body inferior to the head during reconstruction. 
Herein we thus investigate the advantages in imaging performance of including detector modules located superior to the head and angled caudally (i.e., QV) as part of the AdaptiSPECT-C prototype using various ${ }^{123}$ I source distributions. In this design, both primary and scatter photons from regions located inferior to the brain can contribute to SPECT projections acquired by the QV and other detector modules. We chose to restrict our study to ${ }^{123}$ I-labelled tracers since these are widely used clinically for striatal and perfusion imaging procedures. In addition, the low abundant high-energy photons of ${ }^{123} \mathrm{I}$ might further degrade the imaging performance of AdaptiSPECT-C with the QV ring as they are not as attenuated and scattered to the same extent as the $159 \mathrm{keV}$-principal emission of ${ }^{123} \mathrm{I}$ within the body. They might therefore have a higher deleterious contribution to the various detector rings of our system. Note that the findings presented in this study can be translated to other isotopes commonly used in labelling brain SPECT agents, such as ${ }^{99 \mathrm{~m}} \mathrm{Tc}$ and likely ${ }^{111} \mathrm{In}$. First, we performed GATE Monte Carlo (MC) simulation (Jan et al. 2004) to determine the proportion of photons from organs within the head and throughout the body contributing to projections from the different detector rings of AdaptiSPECT-C, as well as from a true vertex view detector. Then, we assessed the sensitivity improvement obtained with the addition of the QV detectors within the imaging volume-of-interest (VOI). Lastly, we compared through phantom studies emulating ${ }^{123}$ I activity distributions, the imaging performance of the AdaptiSPECT-C prototype with and without the QV ring. In addition to MC data, we performed noise-free analytic simulation and reconstruction solely based on ${ }^{123} \mathrm{I}-159 \mathrm{keV}$ imaging. We expect this scenario to lead to similar performance as would be obtained with ${ }^{99 \mathrm{~m}} \mathrm{Tc}$ tracers.

\section{Methods}

\subsection{AdaptiSPECT-C System Prototype}

The AdaptiSPECT-C prototype simulated in this study consists of 23 detector modules built around a $1.0 \mathrm{~cm}$-thick $\mathrm{NaI}(\mathrm{Tl})$ scintillators that are hexagonal in shape with $10 \mathrm{~cm}$ sides. As shown in Figure 1 . AdaptiSPECT-C is arranged along three rings of detector modules; 9 in the caudal ring, 9 in the middle ring, and 5 in the QV ring.

The prototype was designed using the SolidWorks ${ }^{T M}$ (Dassault Systèmes, Waltham, MA) computeraided design package such that all pinholes are focused at the center (i.e., focal point) of a 21-cm diameter spherical VOI. The size of the VOI was sufficiently large to cover the entire brain of $99 \%$ of the population without truncation (Poston 2000, Nillius \& Danielsson 2010). The detectors were positioned such that there was a minimum of $1.0 \mathrm{~cm}$ between the sides of the crystals to allow for crystal-edge treatment and $5 \mathrm{~mm}$ of lead alloy shielding between the modules to reduce inter-module interactions (Auer, De Beenhouwer, Kalluri, Goding, Furenlid \& King 2018). This resulted in a detector surface-to-focal-point distance of $28.7 \mathrm{~cm}$. The 2 -cm thick spherical multi-pinhole collimator was modelled as tungsten alloy. The collimator surface-tofocal-point distance was $17.5 \mathrm{~cm}$. For the purposes of this study, each of the 23 modules was associated to a single knife-edge pinhole, âdaptable in aperture size, with an $80.5^{\circ}$ opening angle.

Apertures were located at a depth of $0.5 \mathrm{~cm}$ from the inner surface of the collimator, resulting in an 18 $\mathrm{cm}$-distance between the center of the apertures to the focal point. This aperture location was determined to result in the lowest amount of scatter and penetration while maximizing sensitivity (Auer, De Beenhouwer, Kalluri, Goding, Furenlid \& King 2018). The aperture acceptance angle was selected such that the projections of the VOI fit onto the detector without truncation while maximizing the detector surface irradiation. A 2-cm thick external shielding, made up of tungsten alloy, was added to enclose the entire collimator-detector module assembly to reduce counts originating outside the VOI from regions such as the liver, or lungs, as illustrated in Figure 1 and 2. Indeed, photons from regions located inferior to the VOI can contribute to projections acquired by each ring of detector modules. Apertures of all rings view activity beyond the head, but the QV apertures view the entire body (Figure 2). Of the three sets of detectors, the QV ring may thus be the one most impacted by activity originating from outside the VOI (i.e., extra-VOI activity). We investigated the origin and impact on reconstruction of such activity, which may create artifacts and degrade overall image quality. For comparison to the QV views in terms of contributions to the projections from various organs within the body, we also designed a version of the AdaptiSPECT-C with no QV apertures or detector modules. In their place, a single detector module and aperture were inserted normal to the vertex axis (i.e., parallel to the top of the head). 


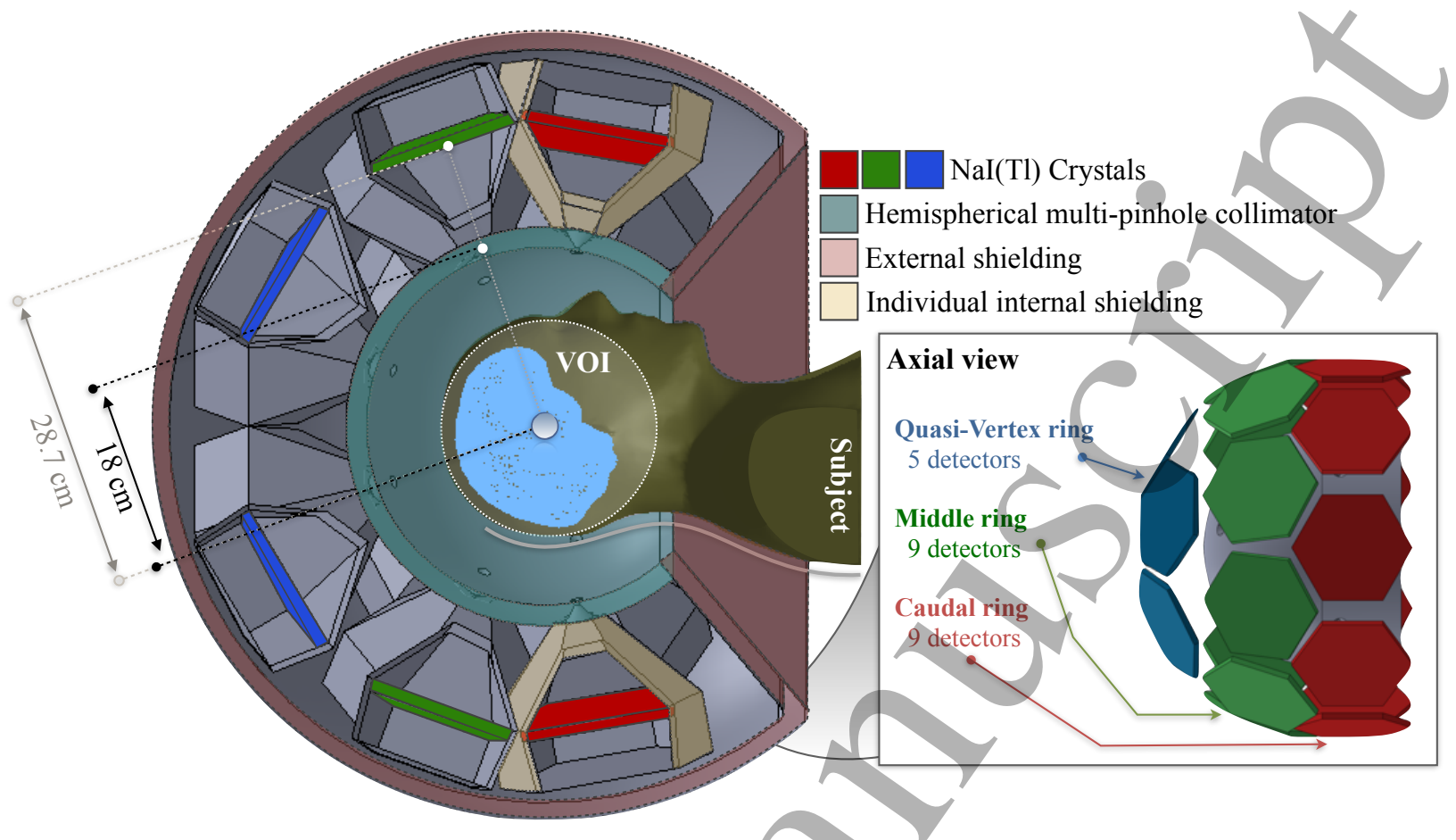

Figure 1: Illustration of the AdaptiSPECT-C prototype geometry, patient positioning, and the volume of interest (VOI) containing the brain. The system consists of three rings of hexagonal detector modules arranged on a spherical surface and irradiated by pinhole apertures set within a spherical multi-pinhole collimator. The three detector rings are color-coded for ease in recognition with the QV ring in blue, the middle one in green, and the caudal one in red.

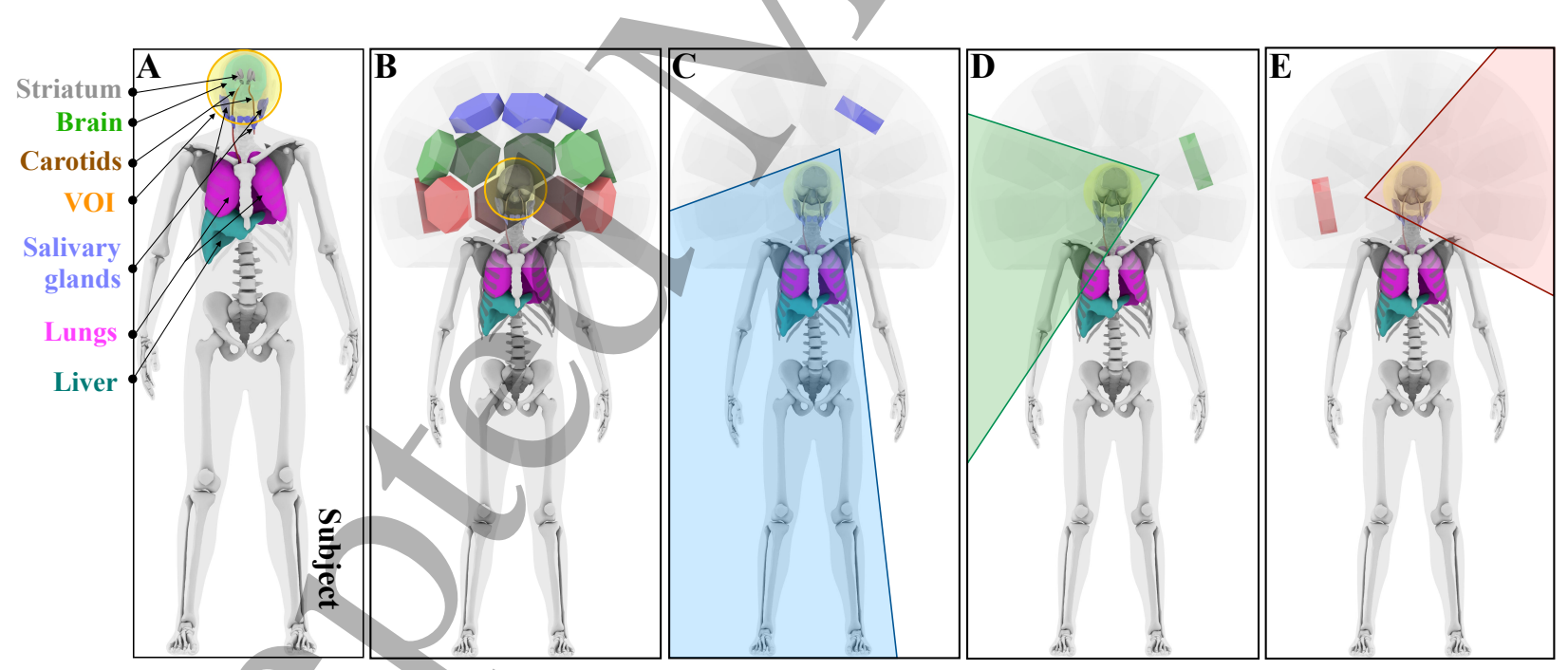

Figure 2: (A) $50^{\text {th }}$ percentile size in men XCAT phantom (Segars et al. 2010) with selected internal organs. (B) The phantom in (A) placed within the AdaptiSPECT-C system consisting of the QV (blue), middle (green), and caudal (red) detector rings. Conical field of view of one sample detector module of the QV (C), middle (D), and caudal (E) rings, respectively. Note that the apertures of all rings view activity beyond the head, but that the QV apertures view the entire body.

\subsection{Monte-Carlo Simulation of the AdaptiSPECT-C using the GATE toolkit}

The AdaptiSPECT-C design created from SolidWorks ${ }^{\mathrm{TM}}$ was converted into an STL ("stereolithography") format for incorporation into the GATE Monte-Carlo simulation toolkit (version 8) (Jan et al. 2004) following 
an approach developed by our group (Auer, Könik, Kalluri, De Beenhouwer, Furenlid \& King 2018). In all our MC simulations, we simulated an extended spectrum of ${ }^{123} \mathrm{I}$ consisting of the $159 \mathrm{keV}$ principal emission plus the 5 most abundant high-energy gamma emissions (i.e., 346, 440, 505, 529, and 539 keV) of 123 $\mathrm{I}$ (Lederer \& Shirley 1978). We refer to this spectrum in the following sections as 'extended spectrum'. For the modelling of down-scatter interactions from ${ }^{123} \mathrm{I}$ low abundance high-energy photons, we considered a compartment behind the 1-cm thick crystal representing photo-multiplier tubes and associated electronics. The back-scattering compartment consisted of a 5 -cm thick single-layer box filled with a mixture of materials as defined in (Rault et al. 2010). The MC simulations modeled all the relevant physics including photo-electric absorption, Compton and Rayleigh scattering, and collimator penetration.

Our previous works suggested that the addition of silicon photomultipliers on the edges of the hexagonal $\mathrm{NaI}(\mathrm{Tl})$ detector in combination with small-diameter high-end photomultiplier tubes at the back of the crystal and with usage of the maximum-likelihood (ML) approach for finding the interaction position and energy could improve the detector performance ( $\mathrm{Li}$ et al. 2017). In accordance with these findings, we therefore modelled in our simulation the intrinsic energy and spatial resolutions of the detector by sampling from Gaussian distributions of $8 \%$ FWHM at $159 \mathrm{keV}$ and $1.5 \mathrm{~mm}$ FWHM, respectively. The Massachusetts Green High-Performance Computing Center (MGHPCC) (https://www.mghpcc.org) was used to perform all the GATE simulations detailed in the following sections.

\subsection{Assessment of Relative Contributions of Activity Throughout the Body to Brain Imaging Using GATE Simulation}

Using MC simulation of the extended cardiac-torso digital anthropomorphic (XCAT) digital phantom (Segars et al. 2010), we determined the relative contribution of activity through the body to projections from the different rings of the AdaptiSPECT-C, as well as from a true vertex view. The low abundant ${ }^{123} \mathrm{I}$ highenergy photons were included in the simulation (i.e., photons $>300 \mathrm{keV}$, which have a total abundance of $\sim 2.5 \%$ abundance). The XCAT phantom enabled definition of the source and attenuation distributions for regions throughout the body for a $50^{\text {th }}$ percentile size in men (Segars et al. 2010), according to PeopleSize anthropometric software (https://openerg.com/psz/index.html), for use in simulation. We simulated acquisition for 30 seconds of an activity concentration of $54 \mathrm{kBq} / \mathrm{cc}$ uniformly distributed within the entire body of the XCAT phantom $(72,827 \mathrm{cc})$. A total of about 100 billion photons were emitted. We determined the number of events detected by AdaptiSPECT-C arising from various anatomical regions of the XCAT phantom from this simulation. This provides an index of the relative contribution of these regions to the AdaptiSPECT-C projections. The voxelized phantom emulating the uniform source distribution consisted of $350 \times 350 \times 1000(2 \mathrm{~mm})^{3}$ voxels. An analysis software based on ROOT libraries (http://root.cern.ch) was developed to determine the primary, scatter, and multiple scatter counts for a typical 15\% energy energy window centered at $159 \mathrm{keV}(147-171 \mathrm{keV})$. Using this program, we estimated the scatter to primary ratio (scatter counts / primary counts), and multiple scatter fraction (multiple scatter counts / scatter counts) for these regions.

GATE does not allow the intersection of dissimilar material objects such as a tungsten collimator and the "air" surrounding the voxelized XCAT attenuation phantom within its bounding "box". Therefore, given the curved nature and proximity to the patient's head of the collimator (Figure 1), the XCAT voxelized attenuation phantom could not be used directly in our MC simulation. These conflicts were resolved by creating and using a mesh representation (STL format) of the XCAT attenuation phantom (Auer, Könik, Kalluri, De Beenhouwer, Furenlid \& King 2018), which describes a given three-dimensional volume with a triangulated boundary surface. The surface described in the STL file is then used in GATE to create a solid volume through the Geant4 G4Tessellated Solid Class, for which all the physics can be simulated (e.g., multiple scattering, etc...) (http://www.opengatecollaboration.org). The STL-based anatomy, consisting of the main structures of the human body, such as the skeleton, brain, lungs and liver, was imported in GATE following a volume-hierarchy approach where each organ is converted to a given predefined material composition. The resulting attenuation map was composed of five different attenuation regions: water, brain, lung, bone, and air. We detailed in a previous work the generation and usage in GATE of our STL-based attenuation phantom (Auer, Könik, Kalluri, De Beenhouwer, Furenlid \& King 2018). Its accuracy in terms of physics modelling was found to be equivalent to that of a regular voxelized phantom, and the results of this study will be published in a future work. 


\subsection{Simulated Phantoms for the Evaluation of Imaging Performance}

A total of four phantoms were used to evaluate the imaging performance of the AdaptiSPECT-C system with and without the QV ring. The uniform sphere and Derenzo phantoms were designed to specifically investigate different aspects of image quality for our prototype system and had an extent limited to that of the system' VOI. Both phantoms were simulated both in an air attenuation medium, and in a 21-cm diameter spherical attenuation medium consisting of water. The air attenuation medium simulation was taken as the reference case as it represents imaging with the negligible effects of attenuation and scatter in the object. The more realistic case consisting of a water medium was then considered for which phantom scatter and attenuation effects were simulated. The uniform and Derenzo activity phantoms and attenuation media were analytically defined in GATE. The other two phantoms modelled striatal and perfusion clinical imaging with ${ }^{123}$ I labeled agents and included the simulation of photons being emitted from the entire body. The extended spectrum of ${ }^{123}$ I including low abundant high-energy photons was modelled in the MC simulation.

Uniform spherical phantom: The 21-cm diameter spherical phantom of uniform activity concentration covering entirely the VOI, was used to study possible contribution of the QV views to the improvement of the sampling of the volume as evidenced by the uniformity of the reconstructed slices (Van Audenhaege et al. 2015). The uniformity was analyzed visually and quantitatively. In addition, the phantom was used to investigate non-uniformity artifacts in reconstructed images (Zanzonico 2008, Van Audenhaege et al. 2015). We simulated acquisition for 15 minutes of an activity of $57 \mathrm{MBq}$ uniformly distributed within the uniform spherical phantom. In this way, with the QV ring, we acquired a total of 37 and 17.8 million detected counts for the air and the water medium simulations, respectively. The corresponding count levels in the absence of the QV ring (i.e., 28.9 and 13.9 million, respectively) were determined based on the relative sensitivities for equal acquisition time and the same activity distribution. This was similarly performed for each study described below.

Derenzo phantom: We simulated a Derenzo phantom to investigate the impact of the addition of the QV ring on tomographic spatial resolution. This cylindrical phantom of $21 \mathrm{~cm}$ diameter by $6 \mathrm{~cm}$ in height, was adapted from the rod region of the ultra-deluxe Jaszczak phantom ${ }^{\mathrm{TM}}$ from Data Spectrum Corporation (http://www.spect.com/products-all.html) so that it fits within the VOI. The cold activity rods were replaced by hot activity rods to transform the Jaszczak phantom into a Derenzo-like phantom. The phantom was positioned axially and centered within the VOI. Activity was solely contained within the rods and no background activity was considered. The phantom consisted of six sets of rod sources with diameters of 11.1, $9.5,7.9,6.4,4.8$, and $3.2 \mathrm{~mm}$. The center-to-center distance between two adjacent rods was equal to two times the rod diameter. An activity of $72 \mathrm{MBq}$ was simulated as acquired for 15 minutes. In this way, we acquired with the QV ring a total of 48.9 and 22.4 million detected counts for the air and the water medium simulation, respectively. The corresponding count levels in the absence of the QV ring were 39.9 and 19.2 million.

Clinical striatal imaging phantom: The first of the clinical distributions derived from XCAT was that of ${ }^{123}$ I-labeled Ioflupane ( ${ }^{123}$ I-FP-CIT, DaTscan ${ }^{\mathrm{TM}}$ ) which is widely used clinically for striatal imaging (Djang et al. 2012). At the typical time of imaging (i.e., four-hour post injection), Sydoff et al. reported that DaTscan activity in the brain is concentrated in the striatum at $3.5 \%$ of the injected dose, liver at $23.6 \%$, lungs at $11.1 \%$, and salivary glands at $0.8 \%$ (Sydoff et al. 2013). This results in activity ratios of $8: 4$ : $1.8: 6.2: 1$ for striatum : salivary glands : lungs : liver : background regions, respectively. The striatum consists of the putamen, the caudate, and the substantia nigra. Since AdaptiSPECT-C was designed to have higher sensitivity than that of a typical dual-headed LEHR parallel-hole system, we investigated the impact of the level of acquired counts on image quality, and the usefulness of the QV ring even for high-count acquisition. We considered two count levels in this study for AdaptiSPECT-C with QV ring. First, the minimum detected count level (1.5 million) recommended by the Society of Nuclear Medicine and Molecular Imaging (SNMMI) (Djang et al. 2012) for a typical DaTscan acquisition. This count level was achieved by simulating an activity of $179 \mathrm{MBq}$ at the time of injection as acquired 4 hours later for approximately 10 min. Second, we simulated a high-count level of 12 million counts obtained by acquiring the same activity 
(i.e., $179 \mathrm{MBq}$ ) for 80 minutes. The corresponding count levels in the absence of the QV ring were 1.2 and 9.2 million, respectively.

Clinical brain perfusion phantom: The second clinical distribution considered was for the brain perfusion imaging agent ${ }^{123}$ I-isopropyl iodoamphetamine (IMP) (Juni et al. 2009). ${ }^{123}$ I IMP perfusion imaging mirrors cerebral blood flow, and thus has a widespread localization primarily in the grey matter as opposed to ${ }^{123} \mathrm{I}$ DaTscan, which concentrates mainly in the striatum. At the typical time of imaging (i.e., one-hour postinjection) the localization is primarily in the brain at $7.0 \%$ of the injected dose, lungs at $14.6 \%$, and liver at $17.0 \%$ (Ito, Sato, Odagiri, Inoue, Shidahara, Suhara, Hatazawa \& Fukuda 2006, Ito, Inoue, Goto, Kinomura, Taki, Okada, Sato, Sato, Kanno \& Fukuda 2006). Activity in the brain was apportioned between grey and white matter at a ratio of 4 to 1 according to observations reported (Ito, Inoue, Goto, Kinomura, Taki, Okada, Sato, Sato, Kanno \& Fukuda 2006). The remaining $61.4 \%$ of injected dose was assumed to be uniformly distributed in all other parts of the body. This results in activity concentration ratios for striatum : grey matter : white matter : lungs : liver : background of $10.1: 9.3: 2.3: 6.0: 11.4: 1$. Similar to the DaTscan distribution, two count levels were considered for the AdaptiSPECT-C with QV ring, a count level of 5.5 million in accordance with the SNMMI recommendation (Juni et al. 2009), and a high-count level of 33.8 million. The corresponding count levels in the absence of the QV ring were 4.2 and 25.4 million, respectively. In this way, the perfusion phantom was simulated with an activity of $175 \mathrm{MBq}$ at the time of injection as acquired 1 hour later for $21 \mathrm{~min}$ and 126 minutes, respectively.
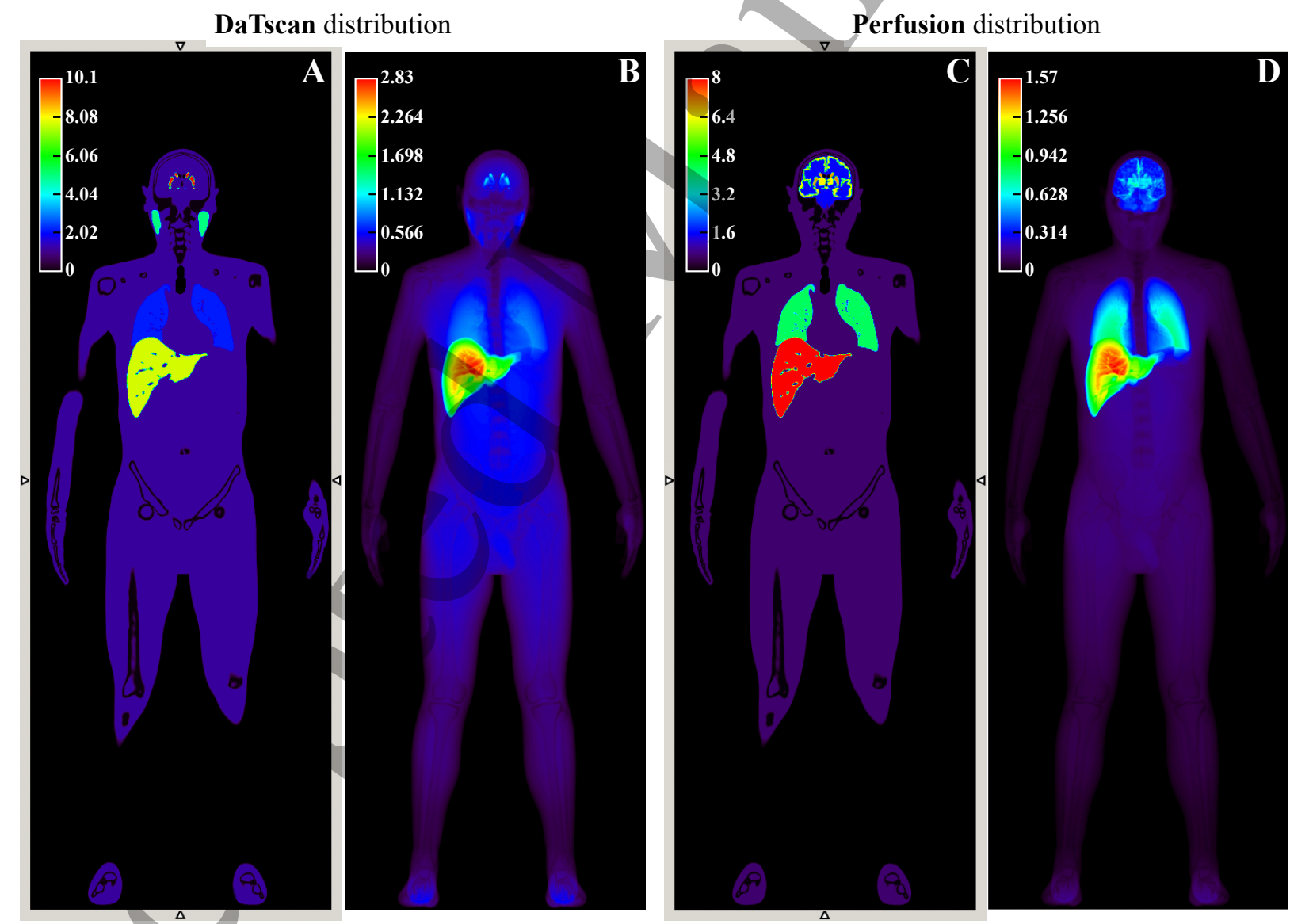

Perfusion distribution

Figure 3: Central coronal slice (A) and average intensity over all the coronal slices (B) of the $50^{\text {th }}$ percentile size in men XCAT phantom emulating normal ${ }^{123}$ I-FP-CIT DaTscan distribution. Central coronal slice (C) and average intensity over all the coronal slices (D) of ${ }^{123}$ I-IMP perfusion distribution for the same phantom size.

The resulting perfusion and DaTscan activity distributions are shown in Figure 3 Similar to the methodology explained in section 2.2, we used an STL-based attenuation phantom that was aligned with 
the voxelized activity distributions represented by an array of $350 \times 350 \times 1000(2 \mathrm{~mm})^{3}$ voxels.

\subsection{Analytic Noise-Free Simulation and Iterative Image Reconstruction}

We developed a generic GPU-accelerated platform for fast analytic simulation and reconstruction of single and multi-pinhole SPECT geometries (Zeraatkar et al. 2016, Auer et al. 2019). The software models the geometric and physical properties of AdaptiSPECT-C (e.g., position and orientation of detectors and apertures, pinhole acceptance angle and size) and the object. We further improved the software by incorporating in addition to the geometrical system response, an analytical modelling of the depth-ofinteraction, the intrinsic spatial resolution, and the detection efficiency (Auer et al. 2019). We also make use of the effective diameter for resolution instead of the physical diameter of the pinhole to take the penetration through the aperture edges into account (Accorsi \& Metzler 2004). The projector and reconstruction software is based on a single ray-tracing approach described in detail in (Zeraatkar et al. 2016). It was demonstrated in this work that the projection into the detector of a given voxel of the image domain passing through the pinhole is circular when the detectors and apertures planes can be considered parallel. The location and the size of the circular projection onto the detector plane can be analytically determined. The sensitivity of the image voxel is then distributed among the projection pixels which belong to the circular projection. For a given object, the global projection is formed by summing up all the individual projections of the voxels defining the object considered. The back-projection step is performed similarly in a reverse process. The image reconstruction was based on the maximum-likelihood expectation-maximization (MLEM) algorithm (Shepp \& Vardi 1982). The analytic software does not model and therefore correct for interactions of the low abundance high-energy photons also emitted by ${ }^{123} \mathrm{I}$ and scatter withín the system. It does however model attenuation within the patient and corrects for it during reconstruction using the attenuation map formed from the XCAT or other phantoms simulated as being imaged. The performance of the analytic projector and the reconstruction software has demonstrated good agreement with MC simulations and MC-based H-matrix reconstruction, respectively (Auer et al. 2019).

As described in section 2.4. for the Derenzo and uniform phantoms, MC projections were simulated in air and water-equivalent attenuation media. For water, projections were reconstructed following a perfect scatter rejection approach, described in (Farncombe et al. 2004). The true scatter distribution obtained by differentiating scatter from primary photons in the simulation, was subtracted from the projection data prior to reconstruction. The primary-only projections were reconstructed with a ray-driven attenuation correction method (Zhao \& Reader 2003). For the DaTscan and perfusion studies, an STL-based attenuation phantom was used to create the MC projections, which was converted into a voxelized phantom for use in attenuation correction following an approach similar to the one described in (Patil \& Ravi 2005). The projections were reconstructed following the perfect attenuation and scatter corrections detailed above.

In addition to the MC dataset, we performed noise-free analytic simulations and reconstruction for the Derenzo, the uniform sphere, the DaTscan, and the perfusion studies with no attenuation modelling and correction using the software described above. In all these analytic simulations and reconstructions solely the imaging of the $159 \mathrm{keV}$ photons of ${ }^{123} \mathrm{I}$ was modelled. The low abundance high-energy photons also emitted by ${ }^{123} \mathrm{I}$ were not simulated in the analytic simulations and reconstructions. This analytic scheme represents the best achievable imaging performance scenario since the forward projection operator used in the reconstruction matches perfectly the analytic simulator employed in generating the noise-free projections. However, since attenuation is not modelled in these simulations, the counts related to extra-VOI regions have a higher impact on reconstruction and quantification, and therefore this scheme represents the worst-case scenario in terms of degradation related to the extra-VOI activity.

A projection matrix of $200 \times 200$ with a $(1.0 \mathrm{~mm})^{2}$ pixel size was used for acquisition by each detector module. To avoid edge effects, potential artifacts (Zeng 2011) and to allow the extra-VOI counts to be reconstructed outside of the VOI, the reconstruction matrix $\left(120 \times 120 \times 120\right.$ by $\left.(2 \mathrm{~mm})^{3}\right)$ and domain (i.e., $24 \mathrm{~cm}$ diameter sphere) were defined larger than the physical dimensions of the VOI. No prior or postreconstruction filter was applied. Reconstruction were performed on a single Nvidia Quadro M4000 GPU equipped workstation, and the computation time was about 1 minute and 45 seconds per iteration (Auer et al. 2019).

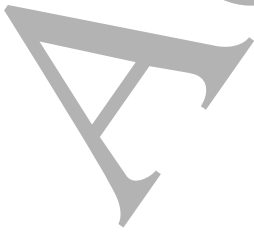




\subsection{Sensitivity measurements}

The projector/reconstruction software described in section 2.5 was used to create 3D maps of relative sensitivity. Two maps were created to assess the impact of the QV-ring detectors on imaging, one with and another without the QV ring. Each voxel of a given sensitivity map reflects the probability of a photon emitted from that voxel to be detected by the 23 (or 18) detector modules of the AdaptiSPECT-C system with (and without) the QV ring. These relative sensitivity values account for the attenuation loss within the patient's head. For display purposes, the maps were confined within the patient's head, i.e., all voxels
outside this region were set to zero.

In addition, through MC simulations, we evaluated the volumetric sensitivity of the system with and without the QV ring for a spherical source corresponding to the target VOI (i.e., 21-cm diameter sphere) uniformly filled with ${ }^{123} \mathrm{I}$ activity. The low abundant ${ }^{123} \mathrm{I}$ high-energy photons were included in the simulation. Two 21-cm diameter spherical attenuation media were considered, one made up of air and one of water. Volumetric sensitivities were computed as the ratio of the total number of detected counts over the number of emitted photons. We then estimated the gain in sensitivity achieved by the addition of the QV ring for AdaptiSPECT-C by comparison with a dual-headed parallel-hole SPECT system (Philips, BrightView). We simulated projections of the 21-cm diameter sphere in air and water attenuation media on the BrightView system using the LEHR-VXHR collimator in accordance with other published works (Goorden et al. 2009, Nillius \& Danielsson 2010) when comparing MPC brain SPECT imaging to dualheaded SPECT imaging. The hole size, the bore length, and the septal thickness of the LEHR-VXHR were 2.03, 54.0, and $0.152 \mathrm{~mm}$, respectively. The simulation model of the BrightView system was validated against experiments in a previous study (Könik, Auer, De Beenhouwer, Kalluri, Zeraatkar, Furenlid \& King 2019).

The sensitivity comparison between the BrightView and AdaptiSPECT-C systems was performed for an equal spatial resolution at the center of the VOI. We computed from (Moore et al. 1992) a spatial resolution of $8 \mathrm{~mm}$ at $13.5 \mathrm{~cm}$ away from the collimator entry face for the BrightView system.

To match this spatial resolution at the center of the VOI, we computed using the pinhole collimator equations from (Goorden et al. 2009) a pinhole diameter of $2.72 \mathrm{~mm}$ for AdaptiSPECT-C. This diameter was used only for sensitivity comparison. In the rest of the paper, taking advantage of the AdaptiSPECT-C adaptive capability, we set the pinhole diameter to $4 \mathrm{~mm}$ to reduce by more than a factor of two the lengthy MC computation time. This was especially important when simulating photons emitted throughout the entire body of the XCAT phantom. The intrinsic resolution and energy resolution were set in MC simulation to $3 \mathrm{~mm}$ and $10 \%$ at $140.5 \mathrm{keV}$, respectively according to the Philips Healthcare 2009 BrightView XCT Technical Specification documentation. For the LEHR-VXHR collimator, 120 equally spaced projections were obtained over $360^{\circ}$ around the $21-\mathrm{cm}$ diameter sphere at a radius of rotation of $13.5 \mathrm{~cm}$. In this way, a total of $58 \mathrm{MBq}$ was simulated as acquired for 30 seconds for the LEHR-VXHR BrightView, and for AdaptiSPECT-C with $2.72 \mathrm{~mm}$ and $4 \mathrm{~mm}$ diameter aperture, in air and water media.

\subsection{Figures of Merit for Assessing Impact of QV Ring on Image Quality and Quantification}

To assess the impact of the QV ring on image quality, in addition to visual assessment of the reconstructions, several figures of merit described in this section were used. One of these was the Normalized-Root-MeanSquare-Error (NRMSE) as a metric of the fidelity with which the source distribution was estimated from the reconstruction. NRMSE was defined as:

$$
\text { NRMSE }=\sqrt{\frac{\sum_{i}\left(I_{R}(i)-I_{G T}(i)\right)^{2}}{\sum_{i} I_{G T}(i)^{2}}}
$$

where $I_{R}, I_{G T}$, and $i$ are the reconstructed image, the ground truth image, and the voxel index, respectively. The source distribution with the same voxel dimensions as the reconstruction was used as the ground truth image. The voxel value of the reconstructed and the ground truth images reflects the total number of photons emitted during the entire acquisition per unit of volume (i.e., voxel) for each phantom.

Since the convergence rates were different for each reconstruction scenario (see section 2.4 and 2.5 ), images were qualitatively compared for the lowest NRMSE values obtained over the number of iterations 
performed. We also estimated from the reconstruction the bias as a measure of quantitative accuracy. Bias was computed as follows:

$$
\operatorname{bias}(\%)=\frac{\left|\overline{I_{R}}-\overline{I_{G T}}\right|}{\overline{I_{G T}}} \times 100
$$

where $\overline{I_{R}}$ and $\overline{I_{G T}}$ denote the voxel value averaged over the phantom-specific region of interest (ROI) of the reconstructed and the ground truth images, respectively. For NRMSE and bias calculation, the ROI was defined as a sphere of $21 \mathrm{~cm}$ diameter corresponding to the system's VOI.

Besides NRMSE, bias, and visual interpretation of phantoms, we performed quantitative analysis on the reconstructions of two clinical distributions to determine if there were regions quantitatively improved by the addition of the QV ring. For both the DaTscan and perfusion studies, activity recoveries (\%AR) were estimated in multiple clinically relevant cerebral regions. To differentiate under from over-estimation of activity, activity recovery in a given ROI was computed as,

$$
\% \mathrm{AR}=\frac{\overline{I_{R}}}{\overline{I_{G T}}} \times 100
$$

For the perfusion study, we generated from the XCAT phantom the following seven 3D ROIs corresponding to the striatum, caudate, putamen, substantia nigra, thalamus, grey matter, white matter and the cortex plus cerebellum. Similarly, the striatum, occipital lobe, caudate, putamen, and substantia nigra were considered for the DaTscan study. No filtering was applied to the reconstructed images for the quantitative analysis to avoid enhancement of the partial volume effect (PVE) (Kessler et al. 1984) on estimation of \%AR.

In addition, the striatal binding ratio (SBR) was computed for quantification of agent localization within the striata (Darcourt et al. 2010, Kuo et al. 2014, Djang et al. 2012). The SBR was computed as follows,

$$
\mathrm{SBR}=\frac{\overline{I(s t r)}-\overline{I(b k g)}}{\overline{I(b k g)}}
$$

where, $\overline{I_{b k g}}$ and $\overline{I_{s t r}}$ denote the average voxel value within the background (i.e., nonspecific binding) and the striatum (i.e., specific binding) ROIs, respectively. In accordance with clinical guidelines, the occipital region was defined as the background region to assess nonspecific binding (Darcourt et al. 2010, Kuo et al. 2014, Djang et al. 2012). The specific binding was estimated for the entire striatum, consisting of the substantia nigra, the putamen, and the caudate. From the SBR values, the \%SBR recovery value was computed as the ratio of the SBR estimated from the reconstruction over the true SBR computed from the ground truth image. Thus, a value of $100 \%$ indicates a perfect match between true and reconstructed values. We reported SBR and activity recovery values closest over iterations to the expected value 100. For the MC simulation with a clinical count level, average value over five noise realizations and standard deviation were considered for the $\% \mathrm{AR}$ and \%SBR.

\subsection{Assessment of the Impact of Counts from extra-VOI Activity on Image Quality with and without the QV-Ring Detectors}

We made use of the DaTscan and perfusion high count MC simulations described in section 2.4. The original emission locations within the entire body of the XCAT phantom were recorded for events acquired by the detectors of AdaptiSPECT-C to assess the impact of extra-VOI activity on image quality. For each source distribution, we considered a first dataset consisting of counts originating only from activity within the VOI, and a second one comprising activity originating from the entire body (i.e., equivalent to the VOI-only scenario plus addition of extra-VOI counts). Each of these datasets was obtained from all three rings of detectors and enabled the comparison of image quality for the entire system when activity was confined to the VOI versus where there was activity throughout the body. To allow comparison of the impact of the presence of the QV ring detectors, an additional set of projections where the QV ring detectors were absent was made from each of the datasets. To facilitate visual comparison, difference images highlighting the spatial distribution and proportion of these counts originating from extra-VOI regions were estimated. These images reflect the absolute difference between the image reconstructed for the VOI only and the one obtained for the whole-body. Since we aimed to investigate the impact of extra-VOI activity on image quality within the VOI, the values of the voxels located outside of the VOI were set to 0 in the difference images. One goal behind the difference image analysis was to evaluate, whether the addition of the QV ring created 
and/or worsened any artifacts related to extra-VOI counts seen on reconstruction. We also investigated the impact of these extra-VOI counts with and without inclusion of the QV ring quantitatively by calculating the lowest NRMSE achieved over iteration for all cases (i.e., analytic noise-free, MC high-count, and MC clinical count levels). Lastly, we report for DaTscan and perfusion distributions the proportion of counts originating from regions located within and outside the VOI, such as brain, grey and white matter, striatum, salivary glands, lungs, and liver. This investigation aimed at determining the region of emission of these extra-VOI counts for clinically relevant activity distributions, which is complementary to the study of section 2.3 performed for a uniform distribution of activity.

\section{Results and Discussion}

\subsection{Relative Contributions from Anatomical Regions and Nature of the Counts for Detector rings of AdaptiSPECT-C}

In Figure 4 we report the results of our investigation using MC simulations to determine the relative contributions of anatomical regions and the nature (i.e., scatter, primary, or multiple scatter) of the counts acquired by each ring of detectors of AdaptiSPECT-C and a vertex view. The regions investigated (i.e., VOI, striatum, salivary glands, thyroid, liver, lungs, whole-body, head plus neck, torso, and inferior body) are illustrated at the top left of the figure. Counts were normalized for each region to an equal activity concentration of $1.17 \mathrm{MBq} / \mathrm{cc}$ of ${ }^{123} \mathrm{I}$ (corresponding to $1.0 \times 10^{6}$ gammas emitted/sec/cc for the extended spectrum of ${ }^{123} \mathrm{I}$ ) to determine the relative count contributions of each anatomical regions. The volumes for each relevant region, shown in the first column of Figure 4 were/derived from the XCAT software. A separate simulation was carried out for a vertex detector with source distributions from the same phantoms. Count contributions were estimated per ring and then normalized to per detector to compare with counts of the single vertex view. The average acquired counts for each set of detectors emanating from the different regions are given in Figure 4. Scatter-to-primary ratio (S/P), as well as the amount of multiple scatter were estimated for all regions, the vertex view, the QV, middle, and caudal rings. A scatter event in this work is defined as a gamma photon undergoing a single or multiple Compton or Rayleigh scattering interactions with matter (i.e., collimator, internal or external shielding, attenuation phantom, and/or back-scattering compartment) prior to detection within the defined energy window (15\% centered at $159 \mathrm{keV})$. A multiple scatter event is a photon that has scattered more than one time and is recorded within the energy window. These events have potentially larger impact than single scatter on image degradation since multiple scatter can cause a substantial loss of spatial information. The primary counts are the non-scattered events that reached the detectors and are counted within the energy window.

From Figure 4 it can be determined that for a uniform activity distributed throughout the body, $11.4 \%$, $20.1 \%, 18.3 \%$, and $15.5 \%$ of the whole-body detected counts on average for a single detector (counts/detector) for the vertex view, QV, middle, and caudal rings are originating from extra-VOI regions. Most of the counts per detector detected by each ring originated from the head plus neck region: $94.3 \%, 86.9 \%, 94.5 \%$, and $98.6 \%$ of the whole-body detected counts for the vertex view, QV, middle, and caudal rings, respectively. Within the counts coming from the head plus neck region, the lowest proportion of extra-VOI counts is obtained for the QV ring (8.1\%), followed by vertex view (11.4\%) and the other rings (middle ring: 13.6\%, caudal ring: 14.3\%). A small proportion of counts is coming from the torso region, $5.7 \%$ (vertex), $13 \%(\mathrm{QV})$, $5.4 \%$ (middle), and $1.4 \%$ (caudal). The highest proportion of counts coming from this region is obtained for the QV ring (two-fold compared to that of the middle ring or vertex view). The caudal ring acquires the lowest proportion of counts coming from the torso region.

Interestingly, for all detector rings and vertex view, despite their more distant locations, the liver and lungs have higher contribution than the thyroid, because of their larger volumes. Nonetheless, in clinical brain imaging for imaging agents labeled with iodine radionuclides a Lugol's solution (sodium perchlorate) is typically administrated to the patient to block uptake of radioactive iodine in the thyroid (Rojas et al. 2013). A non-significant proportion of counts were determined as originating from beyond the torso region (i.e., inferior body), $0.03 \%, 0.07 \%, 0.03 \%$, and $0.01 \%$ of the whole-body total detected counts, for the vertex view, $\mathrm{QV}$, middle, and caudal rings, respectively. For all of the rings and the vertex view, we observed the highest proportion of scatter and multiple scatter counts came from the region beyond the head plus neck. More extraneous head plus neck region activity counts are detected for the QV ring (13\%) than for the other rings 
Physics in Medicine and Biology, Auer et al. 2020
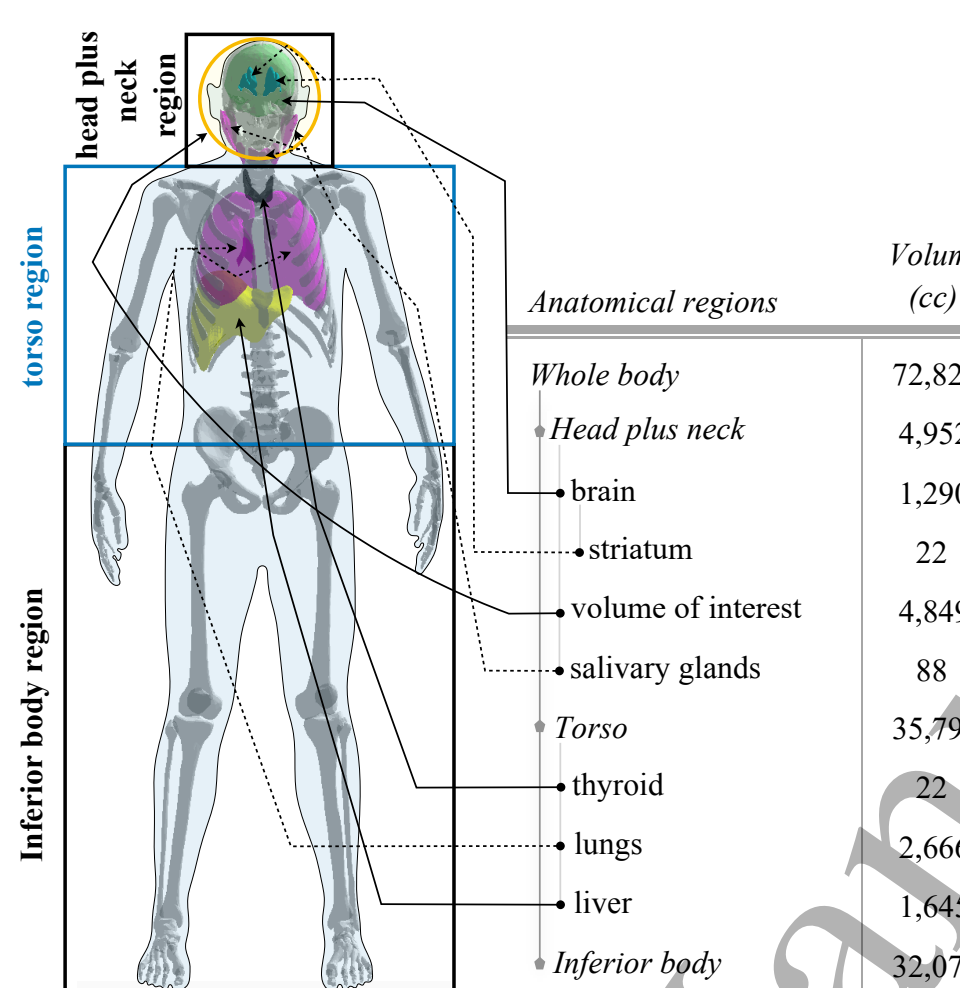

\begin{tabular}{|c|c|c|c|c|c|c|c|c|}
\hline \multicolumn{3}{|c|}{ Middle ring } & \multicolumn{3}{|c|}{ Caudal ring } & \multicolumn{3}{|c|}{ Vertex view } \\
\hline $\begin{array}{l}S / P \\
(\%)\end{array}$ & $\begin{array}{c}\text { Multiple } \\
\text { scatter (\%) }\end{array}$ & Counts & $\begin{array}{r}S / P \\
(\%)\end{array}$ & $\begin{array}{c}\text { Multiple } \\
\text { scatter (\%) }\end{array}$ & Counts & $\begin{array}{l}S / P \\
(\%)\end{array}$ & $\begin{array}{c}\text { Multiple } \\
\text { scatter (\%) }\end{array}$ & Counts \\
\hline 22.9 & 17.4 & 58,093 & 22.1 & 19.0 & 56,857 & 24.7 & 21.7 & 53,174 \\
\hline 21.1 & 16.0 & 54,918 & 20.8 & 15.9 & 56,048 & 22.8 & 19.7 & 50,132 \\
\hline 28.8 & 15.0 & & 31.8 & 15.7 & 14,053 & 27.4 & 16.9 & 23,268 \\
\hline 37.3 & 12.1 & & 42.3 & 16.2 & 184 & 35.2 & 17.3 & 351 \\
\hline 21.5 & 15.8 & 450 & 21.2 & 15.6 & 48,053 & 22.3 & 18.8 & 47,127 \\
\hline 27.9 & 21.7 & & 18.1 & 17.0 & 1,036 & 89.5 & 29.2 & 127 \\
\hline 66.6 & 28.0 & 167 & 400.8 & 64.0 & 789 & 67.7 & 36.3 & 3,027 \\
\hline 45.6 & & 30 & $1,632.1$ & 46.1 & 3 & 164.6 & 39.5 & 5 \\
\hline 112.0 & & 295 & $3,903.6$ & 76.5 & 58 & 204.7 & 45.0 & 158 \\
\hline 545.0 & 142 & 18 & $2,305.1$ & 87.6 & 12 & 274.4 & 52.8 & 22 \\
\hline 2200.0 & & 20 & ++ & 70.6 & 7 & 300.0 & 100.0 & 15 \\
\hline
\end{tabular}

Figure 4: GATE simulation results showing the volume (cc), the scatter to primary ratio S/P (\%), the amount of multiple scatter $(\%)$, and the average counts per second per detector obtained within a $15 \%$ energy window (147-171 $\mathrm{keV}$ ) centered at $159 \mathrm{keV}$. The low abundant ${ }^{123} \mathrm{I}$ high-energy photons were included in the simulation (i.e., photons $>300 \mathrm{keV}, 2.5 \%$ abundance (Lederer \& Shirley 1978)). Counts are shown here per detector for QV, caudal, and middle rings, to be comparable with the single vertex view. Counts were normalized for each region and are here shown for an equal activity concentration of $1.17 \mathrm{MBq} / \mathrm{cc}$ (corresponding to $1.0 \times 10^{6}$ gammas emitted/sec/cc for the extended spectrum of ${ }^{123} \mathrm{I}$ ) to ease comparison between anatomical regions. For the inferior body, no primary counts were recorded for the caudal ring, leading to an infinitely high $\mathrm{S} / \mathrm{P}$ ratio value. This is represented by the '++' symbol. 
and the vertex view $(<6 \%)$. Thanks to the patient body's attenuation, most of these potential extra-VOI counts are absorbed or scattered within the body and thus either not detected or their energy falls outside of the energy window. However, a large proportion of the QV ring counts (87\%) originates from regions within the head plus neck. This ring of detectors can therefore be of interest for improving the sensitivity of AdaptiSPECT-C within the head plus neck region. Indeed, the highest contribution of detected counts per detector from brain structures such as the striatum are obtained for the QV ring (321 counts/cc/detector) and vertex view (351) followed by the middle ring (246). Similarly, this applies to the whole-brain for which we have the following contributions for the vertex view (23,268 counts/cc/detector), QV (21,697), middle $(17,899)$, and caudal $(14,053)$ rings. Moreover, for the brain region, the QV ring has the lowest S/P ratio (27\%), compared to middle $(29 \%)$ or caudal $(32 \%)$ rings, and also the lowest multiple scatter ratio (14.5\%), compared to middle (15\%) and caudal (16\%) rings. Despite that, the QV ring shows better sensitivity per detector for the cerebral region; middle and caudal rings would likely detect more counts since they both consist of nine detectors compared to five for the QV ring. The single vertex detector represents much less of an addition in terms of sensitivity or imaging performance as it is a single detector compared to the five comprising the QV ring.

\subsection{Impact of $Q V$ ring on AdaptiSPECT-C Sensitivity}

We report our volumetric sensitivity results in Table 1 for a water and an air equivalent attenuation medium using the AdaptiSPECT-C and the BrightView systems.

Table 1: Volumetric sensitivity obtained using MC for the dual-headed BrightView system with LEHR parallel-hole collimator and AdaptiSPECT-C with and without the QV ring employing two pinhole diameters $(2.72$ and $4 \mathrm{~mm})$. The gains in sensitivity of AdaptiSPECT-C compared to LEHR-VXHR are shown in parenthesis. We considered two 21-cm diameter spherical attenuation equivalent media (air and water). Spatial resolution values are estimated for a point source at the middle of the VOI from (Moore et al. 1992) for BrightView and from (Goorden et al. 2009, Nillius \& Danielsson 2010) for AdaptiSPECT-C.

\begin{tabular}{lccc}
\hline & $\begin{array}{c}\text { Volumetric } \\
\text { Sensitivity } \\
\text { air } \\
\left(\times 10^{-4}\right)\end{array}$ & $\begin{array}{c}\text { Spatial resolution } \\
\left(\times 10^{-4}\right)\end{array}$ & $\begin{array}{c}\text { at the center of the VOI } \\
(\mathrm{mm})\end{array}$ \\
\hline \hline LEHR-VXHR & 1.54 & 0.816 & 8 \\
\hline AdaptiSPECT-C w/o QV ring $(\mathrm{d}=2.72 \mathrm{~mm})$ & $2.39(\times 1.5)$ & $1.15(\times 1.4)$ & 8 \\
AdaptiSPECT-C w/ QV ring $(\mathrm{d}=2.72 \mathrm{~mm})$ & $3.05(\times 2)$ & $1.47(\times 1.8)$ & 8 \\
AdaptiSPECT-C w/o QV ring $(\mathrm{d}=4 \mathrm{~mm})$ & $4.83(\times 3.1)$ & $2.32(\times 2.8)$ & 11 \\
AdaptiSPECT-C w/ QV $\operatorname{ring}(\mathrm{d}=4 \mathrm{~mm})$ & $6.18(\times 4)$ & $2.97(\times 3.6)$ & 11
\end{tabular}

Assuming a uniform activity distribution filling the target 21-cm diameter spherical VOI, at an equal planar spatial resolution of $8 \mathrm{~mm}$, the volumetric sensitivity of the AdaptiSPECT-C system consisting of all three rings, is approximately two times that of a typical LEHR parallel-hole dual-headed system regardless of the attenuation medium being accounted for or not. As seen in Table 1, the use of a 4-mm diameter aperture for AdaptiSPECT-Cimproves the sensitivity by two-fold. Thus the 4-mm diameter pinhole was used in the rest of the studies to significantly reduce the MC simulation time. We expect to achieve a much higher sensitivity in our finalized prototype as it will consist of additional apertures per detector. Könik et al. demonstrated that in ${ }^{123} \mathrm{I}$ imaging with parallel-hole collimators, approximately one-third of the acquired counts in the $159 \mathrm{keV}$ energy window are related to downscatter interaction of the low abundance high-energy photons of ${ }^{123}$ I (Könik, Auer, De Beenhouwer, Kalluri, Zeraatkar, Furenlid \& King 2019). Since these down-scattered photons degrade image quality, they represent an undesirable contribution to sensitivity. Such counts are in a much lower proportion in multi-pinhole systems. When the volumetric sensitivity was analyzed on a per ring-basis, the contribution of each ring for the spherical activity on the total AdaptiSPECT-C sensitivity, is $21.8 \%, 39.1 \%$, and $39.1 \%$ for the $\mathrm{QV}$, middle and caudal rings, 
respectively. Thus, we determine that the QV ring does make an important contribution to the sensitivity of the system.
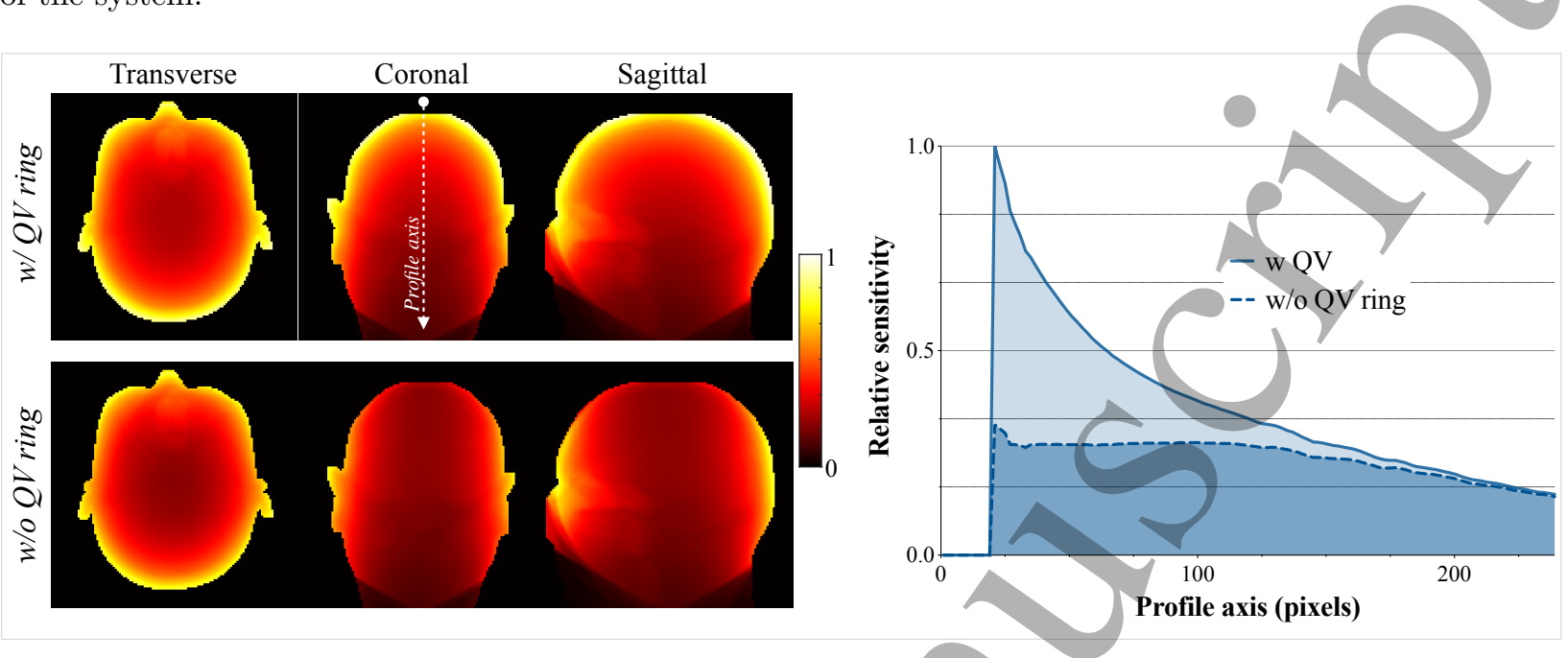

Figure 5: (left) Sample transverse, coronal, and sagittal slices of the sensitivity maps for which attenuation was modelled with (top) and without (bottom) the QV ring. Each voxel value represents the relative sensitivity (i.e., ratio of the detected counts over the emitted counts) summed for all the 23 (18 without the QV ring) heads. Images are shown for the same color scale to enable visual comparison. (right) Sensitivity profiles along the central coronal slice in the axial direction from vertex to caudal (as ińdicated by the white arrow in the top coronal slice), obtained with and without the QV ring.

In Figure 5 we show transverse, coronal, and sagittal slices of the sensitivity as a ratio of detected versus emitted photons per voxel as a function of location in the head of the XCAT phantom in presence of attenuation with and without the QW ring. For a uniform source filling the entire head in presence of attenuation, the ring contributions to the system sensitivity are approximately equivalent to the spherical source used above: $21.5 \%, 39.2 \%$, and $39.3 \%$ for the QV , the middle, and the caudal ring. As both slices and sensitivity profiles in this figure indicate, the improvement in system's sensitivity with the addition of the QV-ring is achieved mainly for the superior region of the head. This gain in sensitivity could help improve detection of perfusion defects, as needed for eârly stage of Alzheimer's disease which usually starts in the superior region of the brain (Matsuda et al. 2007, Kogure et al. 2000). In addition, the superior central regions of the brain (e.g., striatum) are of the utmost interest in DaTscan imaging. The addition of the QV ring provides an overall improvement in uniformity of sensitivity in transverse slices (Figure 5), especially in the superior portion of the head where the cerebrum is located. However, a degradation of the axial uniformity can be observed for the coronal and sagittal slices with the addition of the QV-ring. This is demonstrated by the 4-fold increases in the maximum of the coronal profile of the sensitivity image (right of Figure 5). It was reported that a uniform signal-to-noise ratio (SNR) for the entire VOI is desirable (Van Audenhaege et al. 2015). Non-uniformity in SNR generally results in different noise characteristics that will be visible in uniform phantom reconstructions. This effect will be investigated in the next section.

\subsection{Impact of QV ring on Uniform Activity Reconstruction within the Imaging VOI}

In Figure 6, we present our results of the reconstruction of a 21-cm diameter uniform sphere filling the VOI. The three imaging scenarios considered were noise-free analytic simulation/reconstruction without attenuation modelling and correction (Analytic), MC high-counts simulations/reconstructions in an air medium ( $\mathrm{MC}$ (air)), and in a 21-cm diameter spherical water equivalent medium with attenuation and perfect scatter correction (MC (water)) (see section 2.5 for details). We aimed to visually assess possible artifacts related to insufficient angular and/or axial sampling, and/or non-uniformities in reconstruction as it is usually performed in the clinic with uniformity phantoms (Van Audenhaege et al. 2015). No visual difference was observed in the reconstruction between the case for which the QV ring was present and the one for which it was not, for all scenarios (Figure 6-A). Moreover, the addition of the QV ring does not 
introduce non-uniformities or any other type of artifacts into the reconstruction.

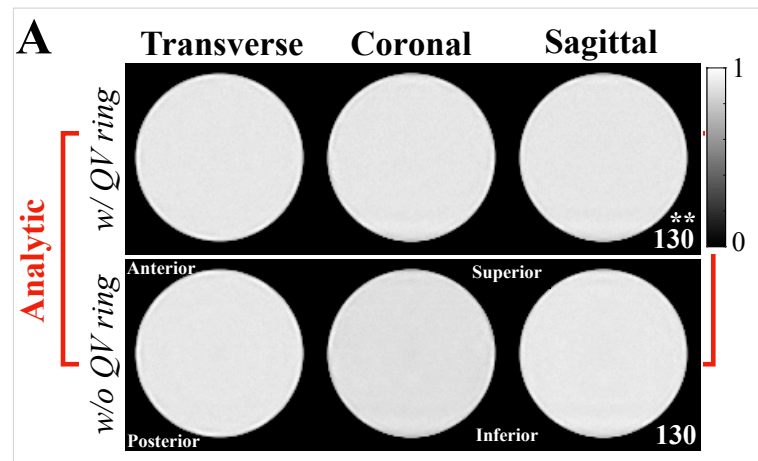

B
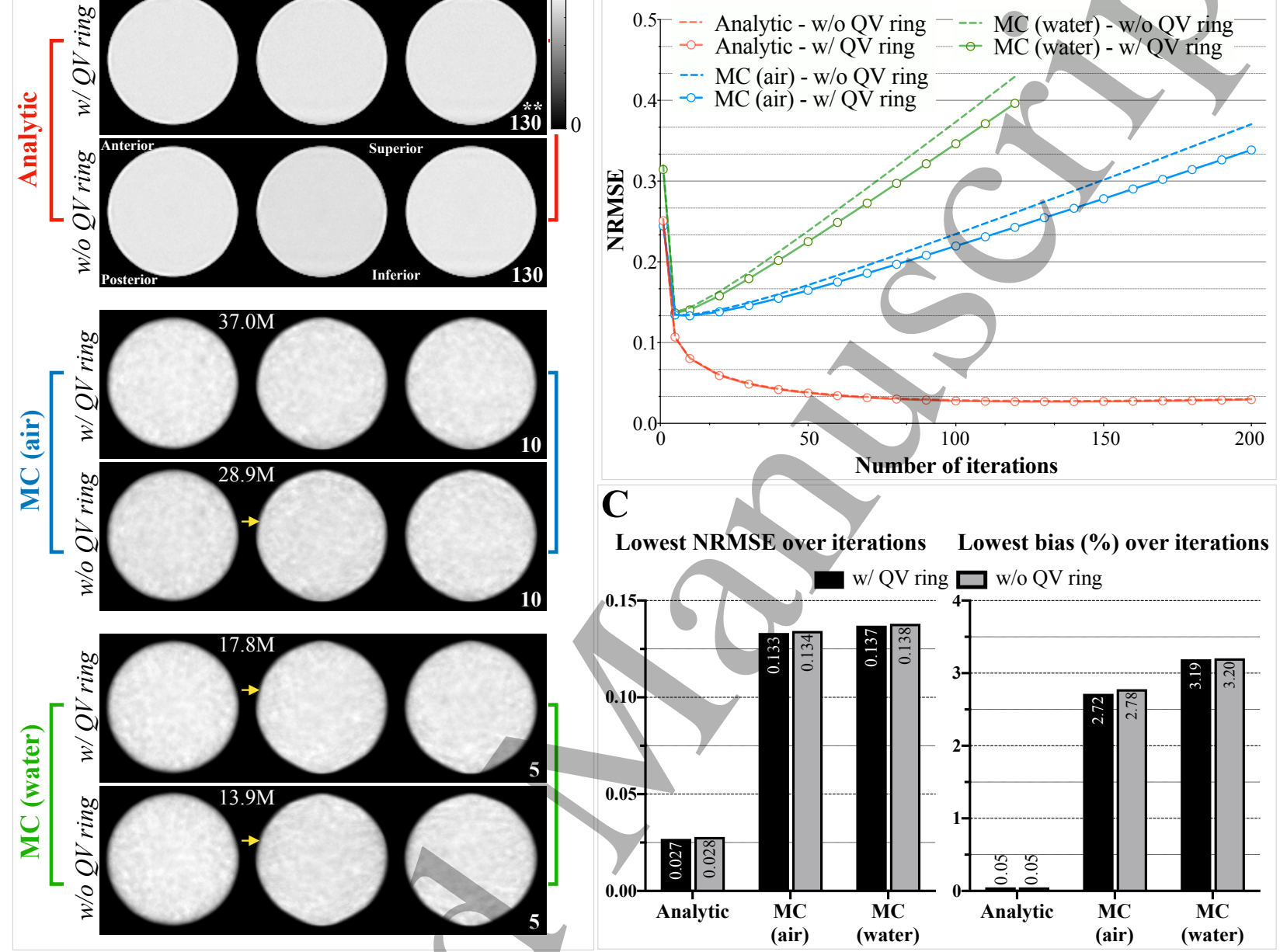

Lowest NRMSE over iterations Lowest bias (\%) over iterations
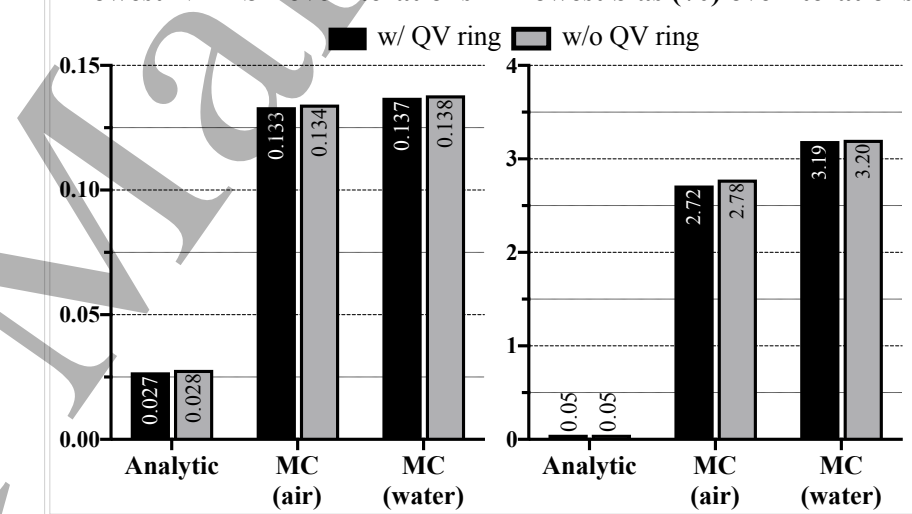

Figure 6: (A) Sample transverse, coronal, and sagittal slices of reconstructed images of the uniform phantom at the iteration showing the lowest NRMSE as provided at lower right (**) on the sagittal slice. Starting from the top the slices are for the noise-free analytic, MC in an air attenuation medium, and then water attenuation medium, scenarios with and without the QV ring. The yellow arrows highlight the streaks artifacts discussed in section 3.3 . The top of the coronal and sagittal slices correspond to the superior side of the VOI. The count levels (M for million detected counts) for each MC scenarios are shown at upper right on the transverse slices. (B) NRMSE values over iterations for all the investigated scenarios with and without the QV ring. (C) Lowest NRMSE and bias values over iterations for all the investigated scenarios with and without the QV ring.

Visually, uniformity is similarly recovered with and without the QV ring for all scenarios. However, streaks artifacts are visible on the coronal slices for the MC reconstructions, whether the QV ring is present or not (see yellow arrows in Figure 6). Those patterns are likely due to inaccuracy in point spread function (PSF) modelling, statistical noise increase, and imperfect attenuation and scatter corrections. These artifacts are slightly visible for the MC air medium reconstruction, but indeed increased for the water equivalent medium. Note that these artifacts are reduced by the use of the QV ring because of the additional counts and sampling it provides. The noise-free analytic scenario represents the reference scenario for which we did not model and correct for attenuation, as well as for ${ }^{123} \mathrm{I}$ high-energy contribution. We considered also no statistical noise in the data, and a perfect match in PSF modelling between projection and reconstruction. Given that this scheme solely modelled ${ }^{123} \mathrm{I}-159 \mathrm{keV}$ photons imaging, its performance can be considered equivalent to those of ${ }^{99 \mathrm{~m}}$ Tc imaging. For those reasons, the best image quality, as well as the lowest NRMSE and bias values are achieved for this scenario. Note, that the streak artifacts are not visible for the analytic scheme. 
The NRMSE and bias are degraded when MC data are used. The inclusion of attenuation and scatter in the simulation results in a slightly larger degradation of the NRMSE compared to the analytic and the MC air medium scenarios. A decrease of total detected counts, downscatter interaction of ${ }^{123}$ I high-energy photons, and inaccuracy in our attenuation/scatter correction are responsible for this deterioration. Quantitively, for all scenarios, small NRMSE and bias improvements are obtained with the QV addition (Figure 6-B and C).

\subsection{Assessment of the Impact of QV ring on Spatial Resolution using a Derenzo Phantom}

In Figure 7, we report our results on the Derenzo phantom study for the three scenarios described in section 2.5 (i.e., Analytic, MC (air), and MC (water)).

A
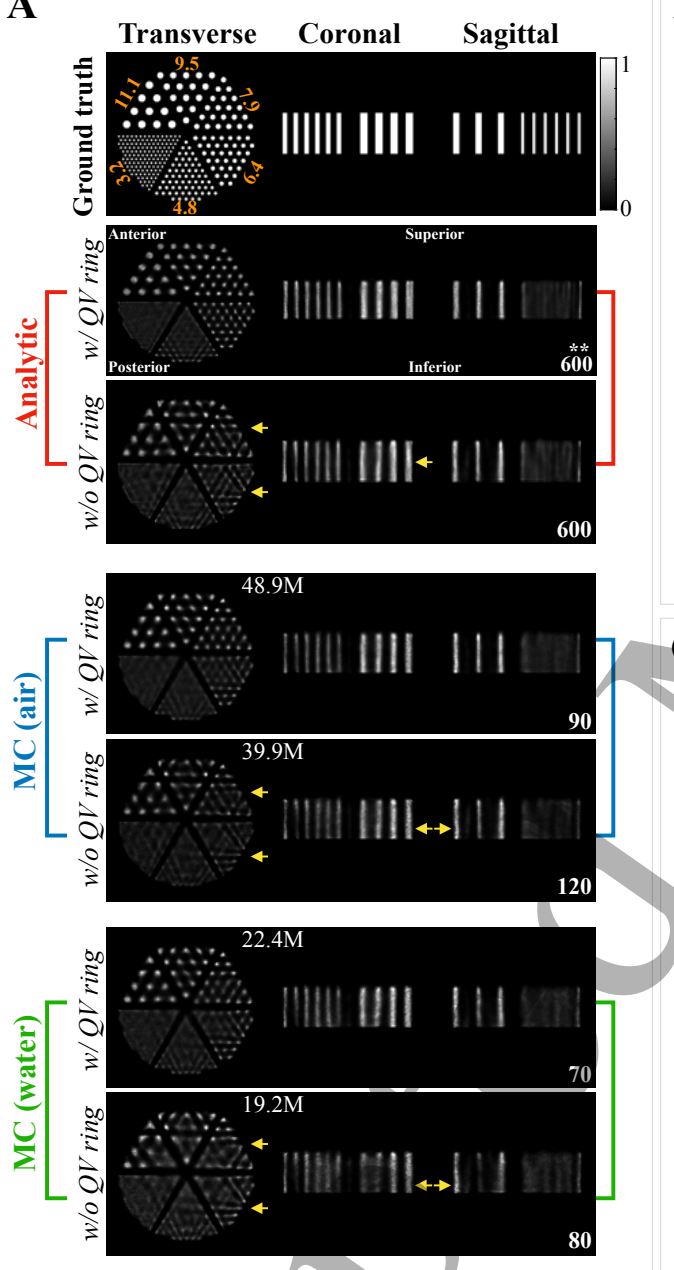

B

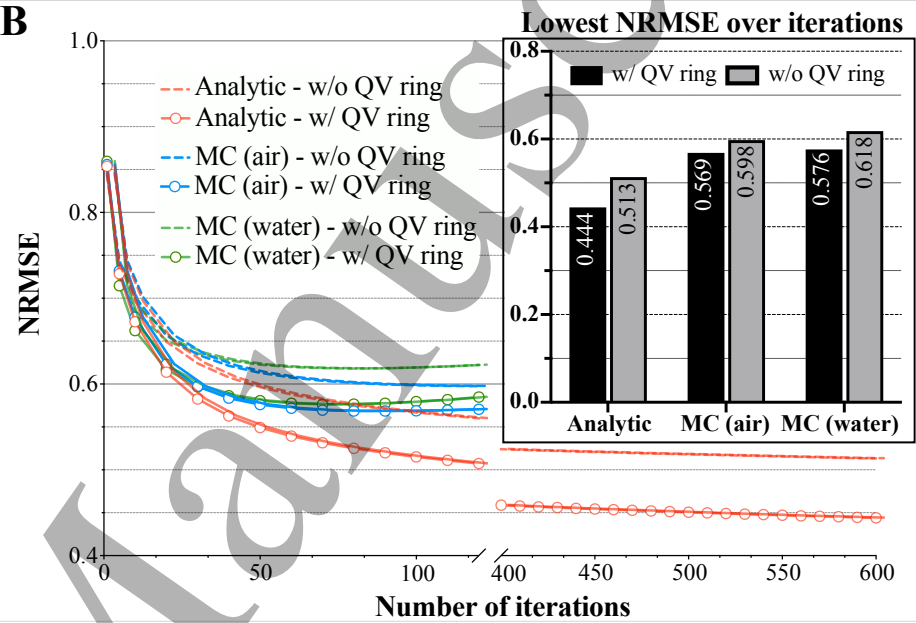

\section{$C_{80}$}

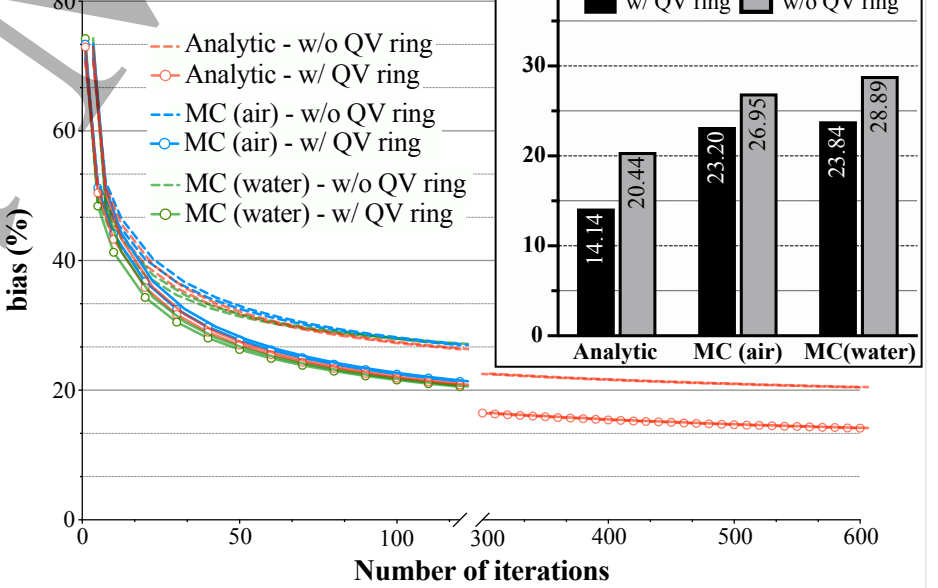

Figure 7: (A) Sample transverse, coronal, and sagittal slices of the reconstructed images of the Derenzo phantom at the iteration showing the lowest NRMSE as provided at lower right $(* *)$ on the sagittal slice. Starting from the top the slices are for the ground truth image and then the imaging scenarios of noise-free analytic, MC in an air and in a water attenuation medium, with and without the QV ring. Hot-rod diameters in $\mathrm{mm}$ are displayed in orange color onto the ground truth transverse slice. Yellow arrows show regions of degradation when the QV ring is absent. The top of the coronal and sagittal slices corresponds to the superior side of the VOI. The count levels (M for million detected counts) for each MC scenarios are shown at upper right on the transverse slices. (B) Plot of NRMSE values over iterations for the three investigated scenarios with a bar-graph showing the lowest NRMSE values with and without the QV ring. (C) Plot of bias values over iterations for the three scenarios with a bar-graph showing the lowest bias values with and without the QV ring. As no local minimum values were found for the analytic scenario over the iteration range considered, we showed the bias values from 1 to 600 iterations. 
We aimed to assess possible improvement in spatial resolution visually and in terms of its impact quantitatively on the NRMSE and bias with the QV ring addition.

Visually noticeable improvement in spatial resolution (i.e., appearance as well as separation of the rods) can be seen in the slices with the addition of the QV ring. For the analytic noise-free data, the $6.4 \mathrm{~mm}$ rod sector could best be resolved when the QV is present. Note that even with sufficient geometric resolution the $3.2 \mathrm{~mm}$ rod segment would have been unresolvable without aliasing artifacts with a $2 \mathrm{~mm}$ reconstruction voxel size, since this voxel size is greater than half of the separation between two rod centers, as stated by the Nyquist-Shannon sampling theorem (Nyquist 1928, Shannon 1949). The absence of the QV ring results in significant artifacts in the slices due also to reduced angular sampling. Indeed, as pointed by arrows in Figure $7 \mathrm{~A}$, line pattern artifacts can be seen visually on the slices and the rods do not appear circular. Rod sectors with diameters below $6.4 \mathrm{~mm}$ are not resolved over the entire segment even by the noise-free analytic scenario for which the best imaging performance is achieved. Given that the analytic scheme solely modelled ${ }^{123} \mathrm{I}-159 \mathrm{keV}$ photons imaging, its performance can be considered a good approximation to that of ${ }^{99 \mathrm{~m}} \mathrm{Tc}$ imaging. The use of MC data in reconstruction degrades the recovery of the $6.4 \mathrm{~mm}$ sector compared to analytic simulation due to statistical noise, and inaccuracy in PSF modelling between MC simulation of projections and analytic reconstruction. The recovery of the $6.4 \mathrm{~mm}$ sector illustrates that the reconstructed tomographic spatial resolution is better than the spatial resolution of $11 \mathrm{~mm}$ computed from the collimator equation at the center of the VOI (Goorden et al. 2009, Nillius \& Danielsson 2010). The spatial-resolution compensation used in this work was based on incorporating the PSF into the system matrix employed in reconstruction, for which case the effect of the system PSF was partially reversed. Such distance-dependent resolution correction can therefore further enhanced image resolution since it acts like a partial deconvolution of the image domain during the iterative reconstruction (Erlandsson et al. 2012). This was similarly reported by Van Audenhaege et al. (Van Audenhaege et al. 2013), who determined that the resolution compensation in iterative reconstruction allowed them to visualize $4 \mathrm{~mm}$ hot-rods even when their target resolution was 6 $\mathrm{mm}$ at the center of their VOI. Further spatial resolution improvement is achieved along the axial direction (coronal and sagittal views) with the QV ring present when GATE data are used and as the level of counts in the projection decreases. The largest visual difference compared to the case for which the QV ring is absent is obtained for the realistic case MC (water), for which gain in sensitivity and sampling plays a major role as the level of counts is the lowest.

Quantitively, NRMSE values are improved by $13.4 \%, 4.8 \%$, and $6.8 \%$ when the QV ring is present for the analytic, MC (air), and MC (water) scenarios, respectively. Bias values are similarly improved by $30.7 \%, 13.7 \%$, and $17.6 \%$ by the QV ring inclusion for the analytic, MC (air), and MC (water) scenarios, respectively.

\subsection{Impact of QV ring on Image Quality for Simulated Clinical Distributions}

We investigated the impact of the QV ring on image quality and quantification for DaTscan and perfusion distributions emulating clinically relevant acquisitions for the three scenarios defined in section 2.4 and 2.5 . noise-free Analytic, MC high-count level (MC-HC), and MC clinical-count level (MC-CC).

\subsubsection{Origin of the counts detected in the $Q V$, Middle and Caudal detector rings}

Table 2 shows the contribution of each detector ring in terms of percentage of total number of detected counts within a $15 \%$ energy window centered at $159 \mathrm{keV}$ for relevant anatomical regions estimated for simulated DaTscan and perfusion source distributions. This table presents complementary results to those of Figure 4 for which we considered a non-realistic uniform activity distributed throughout the body as well as the proportion of counts per detector. From the Table $2,18.1 \%$ and $10 \%$ of the total counts are originating from extra-VOI activity, for DaTscan and perfusion distributions, respectively. For DaTscan, among the extra-VOI counts, $11.7 \%$ come from the head plus neck region, and $6.4 \%$ from regions located beyond. The middle ring has the highest proportion of extra-VOI counts ( $7.3 \%$ of total detected counts), followed by caudal (5.9\%) and QV (4.9\%) rings. Most of the extra-VOI counts originating from regions beyond the head plus neck region contribute to the QV ring (3.4\% of total counts), followed by the middle $(2.4 \%)$ and the caudal (0.6\%). Interestingly, only $37.9 \%$ of the total counts come from the brain, with a contribution of $27.4 \%, 40.9 \%$, and $31.7 \%$ for the QV, middle, and caudal rings, respectively.

The salivary glands, representing $5 \%$ of total counts, are mostly detected by the caudal ring $(62.7 \%)$, 
Table 2: Percentage of total detected counts in all the detectors of the indicated ring (sum of primary and scatter counts) within a $15 \%$ energy window centered at $159 \mathrm{keV}$ for selected anatomical regions obtained for simulated DaTscan (left) and perfusion (right) activity distributions. Volume of each region (cc) is shown in the second column of the table. The region named beyond shoulders represents the torso and the inferior body regions shown on the right in Figure 4

\begin{tabular}{|c|c|c|c|c|c|c|c|c|c|}
\hline \multirow[b]{2}{*}{ Anatomical regions } & \multirow[b]{2}{*}{$\begin{array}{c}\text { Volume } \\
\text { (cc) }\end{array}$} & \multicolumn{3}{|c|}{ DaTscan } & \multirow[b]{2}{*}{ total } & \multicolumn{3}{|c|}{ Perfusion } & \\
\hline & & $\begin{array}{l}\text { QV } \\
\text { ring }\end{array}$ & $\begin{array}{l}\text { Middle } \\
\text { ring }\end{array}$ & $\begin{array}{c}\text { Caudal } \\
\text { ring }\end{array}$ & & $\begin{array}{l}\text { QV } \\
\text { ring }\end{array}$ & $\begin{array}{c}\text { Middle } \\
\text { ring }\end{array}$ & $\begin{array}{c}\text { Caudal } \\
\text { ring }\end{array}$ & total \\
\hline Whole-body & 72,827 & 22.7 & 39.1 & 38.2 & 100 & 24.9 & 40.1 & 35 & 100 \\
\hline Head plus Neck & 4,952 & 19.3 & 36.7 & 37.6 & 93.6 & 22.5 & 38.4 & 34.6 & 95.5 \\
\hline Volume of Interest & 4,849 & 17.8 & 31.8 & 32.3 & 81.9 & 21.8 & 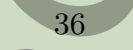 & 32.2 & 90 \\
\hline Brain & 1,290 & 10.4 & 15.5 & 12 & 37.9 & & 33 & 26.5 & 80.9 \\
\hline Grey matter & 664 & 4.8 & 7.4 & 6 & 18.2 & & 20.4 & 17.1 & 50.2 \\
\hline Striatum & 22 & 1.1 & 1.5 & 1.2 & & & 1 & 0.8 & 2.6 \\
\hline White matter & 626 & 5.6 & 8.1 & 6 & 19.7 & .7 & 12.6 & 9.4 & 30.7 \\
\hline Salivary glands & 88 & 0.3 & 1.6 & 3.2 & 5.1 & 0 & 0.2 & 0.5 & 0.7 \\
\hline Beyond shoulders & 67,874 & 3.4 & 2.4 & 0.6 & & 2.4 & 1.7 & 0.4 & 4.5 \\
\hline Lungs & 2,666 & 0.5 & 0.4 & 0.1 & & 0.8 & 0.7 & 0.1 & 1.6 \\
\hline Liver & 1,645 & 0.3 & 0.1 & 0.1 & & 0.3 & 0.1 & 0.1 & 0.5 \\
\hline
\end{tabular}

followed by the middle (31.4\%) and much less by the QV (5.9\%). Since this region is partly contained within the VOI, a scaling artifact of the striatal region might be observed. Indeed, in DaTscan imaging, the intense uptake of the salivary glands may result in the striatal uptake being scaled down (Banks et al. 2019). We will investigate in the following section if such artifacts can be seen in our reconstruction.

Similar findings can be highlighted for perfusion, despite a lower proportion of extra-VOI counts compared to DaTscan, approximately $22 \%, 36 \%$ and $32 \%$ of the VOI counts contribute to the QV, the middle, and the caudal ring, respectively. Most of the counts originate from the head plus neck region (95.5\% of total counts), with slight contribution of regions located beyond it (4.5\%). The extra-VOI activity contributes mostly to the middle (4.1\% of total counts) and the QV (3.1\%) rings, but less to the caudal $(2.8 \%)$. Most of the extra-VOI activity originates from regions within the head plus neck (5.5\% of total counts), the rest come from regions beyond the shoulders (4.5\%) such as the lungs (1.6\%) and liver (0.5\%). Regions located beyond the head plus heck contribute predominately to the QV ring (2.4\% of total counts) and the middle (1.7\%), but less to the caudal (0.4\%). The QV ring detects only $24.2 \%$ of the VOI counts as compared to the middle and the caudal rings with contributions of $40 \%$ and $35.8 \%$, respectively.

For both DaTscan and perfusion, the QV ring shows improved sensitivity for regions located in the superior area of the brain, such as the striatum, which contributes at $28.9 \%$ (30.8\% of striatum counts), $39.5 \%(38.4 \%)$, and $31.6 \%$ (30.8\%) to the QV, middle, and caudal rings, for DaTscan (for perfusion), respectively. Interestingly, for the striatum, the QV ring has an equivalent detection to that of the caudal ring. Hence, for superior region of the brain, the ratio of counts detected per detector, is higher for the QV ring than for the other rings, since it consists of only five versus nine detectors for the two other rings.

\subsubsection{Impact on Image Quality of the Extra-VOI counts for Imaging With and Without the QV ring}

Figure 8 shows select transverse, coronal, and sagittal slices of the difference images of the DaTscan and the perfusion reconstructions at the lowest NRMSE obtained for the MC-HC scenario with and without the QV ring addition (see Section 2.8 for details). Each voxel represents the absolute difference between the images reconstructed from the dataset containing only counts originating within the VOI and images reconstructed using events that originated throughout the body. The value of voxels located outside of the VOI was set to 0 . These difference images thus illustrate regions within the VOI impacted by extra-VOI activity. For both distributions, small differences related to extra-VOI counts are introduced in the brain regions by the addition of the QV ring as compared to when it is absent. Image quality degradation occurs mostly at the 
A
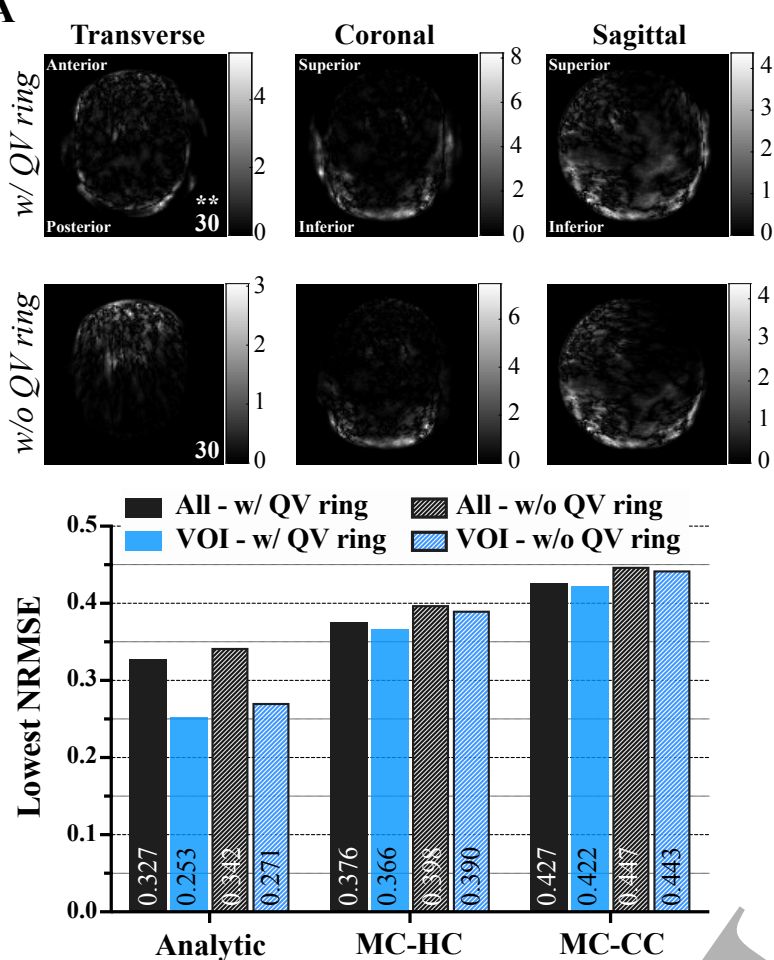

B
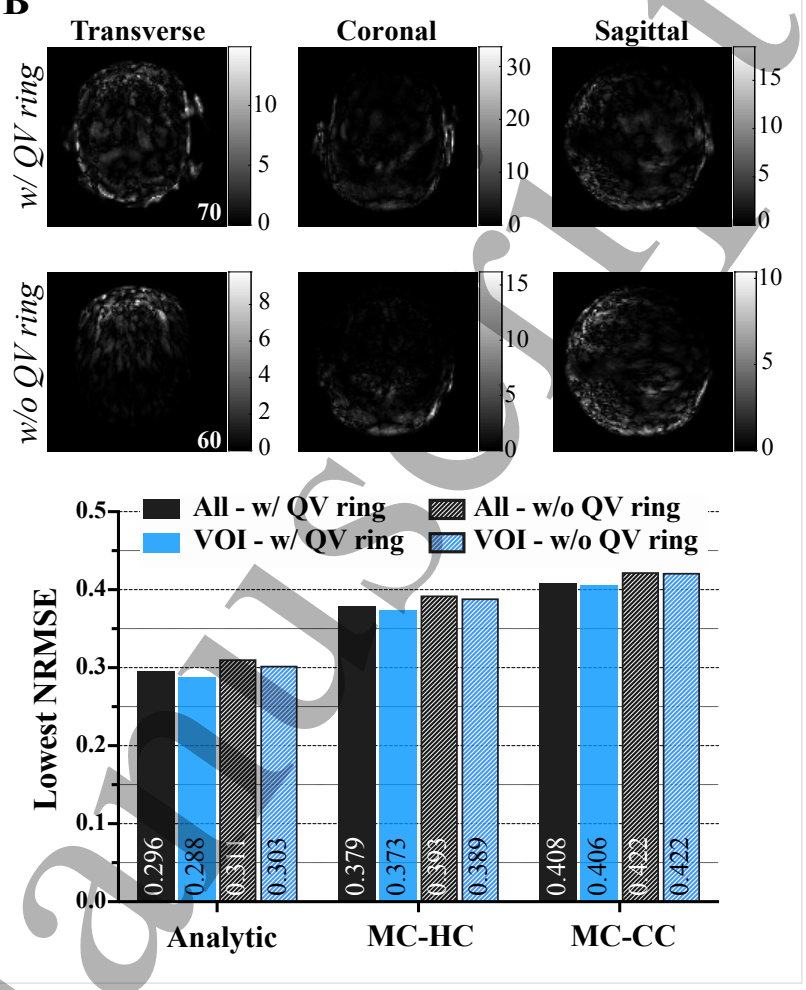

Figure 8: (top) Sample transverse, coronal and sagittal slices of the difference images between with and without extra-VOI activity for the DaTscan (A) and perfusion (B) phantoms showing the lowest NRMSE as provided at lower right on the transverse slice $\left(^{* *}\right)$ obtained for the $\mathrm{MC}$ high-counts scenario with (top row) and without (bottom row) the QV ring. Difference images show the spatial locations of regions impacted by extra-VOI counts. Image scales are in $10^{4}$ emitted photons and are indicated for each slice. The top of the coronal and sagittal slices correspond to the superior side of the VOI. (bottom) The lowest NRMSE values over iterations for counts from the whole-body (All) and VOI-only counts (VOI) obtained for the noise-free Analytic, MC high and MC clinical count level scenarios with and without the $\mathrm{QV}$ ring.

caudal regions of the coronal and sagittal slices, as well as the edges of the transverse slices. In Section 3.5.1 we demonstrated that most of the extra-VOI counts contribute to the middle $(7.3 \%$ of total counts for DaTscan and $4.1 \%$ for perfusion) and caudal rings (5.9\%, $2.8 \%)$, as opposed to the QV ring $(4.9 \%, 3.1 \%)$. Thus, some differences related to extra-VOI counts can be seen even when the QV ring is not included. Nevertheless, the QV ring slightly increases the impact of such counts on the reconstruction, mostly at the edges of the VOI. To help reduce the addition of extra-VOI counts into clinically relevant regions (i.e., the brain), we have employed a volume for reconstruction larger than the physical dimension of the VOI (i.e., 24-cm diameter sphere). Despite a two-fold increase in extra-VOI counts contribution compared to perfusion, equivalent differences in terms of numbers, shapes and scales are seen on the reconstructions for DaTscan.

At the bottom of Figure 8 we show the lowest NRMSE values over iterations using VOI-only counts and then using all counts including extra-VOI counts in reconstruction for the three imaging scenarios (i.e., Analytic, MC-HC, and MC-CC) with and without the QV ring. The noise-free analytic scenario for which no attenuation was modelled, shows the highest degradation in NRMSE when the extra-VOI counts are included. Understandably, this scenario represents the worst case since extra-VOI counts are not attenuated by the patient's body. Interestingly, even for the noise-free scenario, the addition of the QV ring leads to an NRMSE improvement of $4.4 \%$ and $4.8 \%$ for DaTscan and perfusion, respectively, likely due to the improved sampling. The enhancements are about $(5.5 \%, 3.6 \%)$ and $(4.5 \%, 3.3 \%)$ for the MC high and clinical count levels, respectively. The analytic scenario shows larger NRMSE degradation (30\%) when extra-VOI counts are added for DaTscan than for perfusion, since more extra-VOI counts are present in the data. This deterioration is much less for the MC high-count scenario (2.5\%), and even less for the MC clinical 
count level scenario (1\%). Since we have fewer extra-VOI counts for perfusion, the NRMSE is degraded by $\sim 3 \%$ when extra-VOI counts are included. For the clinical MC scenarios, the respective deteriorations are about $(\sim 1.5 \%)$, and $(0.25 \%)$ for a high and a clinical count level. Indeed, most of the extra-VOI counts are attenuated for the MC scenarios. As the number of acquired counts decreases, the effect of the statistical noise becomes predominant as compared to the impact of the extra-VOI counts on reconstruction. In the following sections, to emulate clinical imaging, the entire datasets consisting of counts emitted throughout the body were used for reconstruction and quantitative analysis.

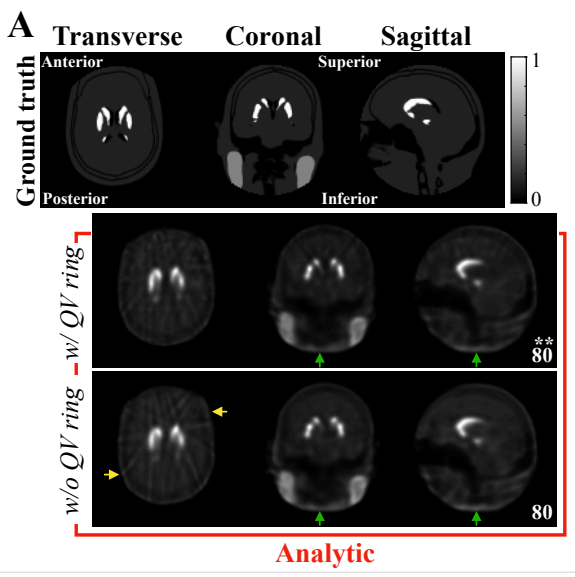

B

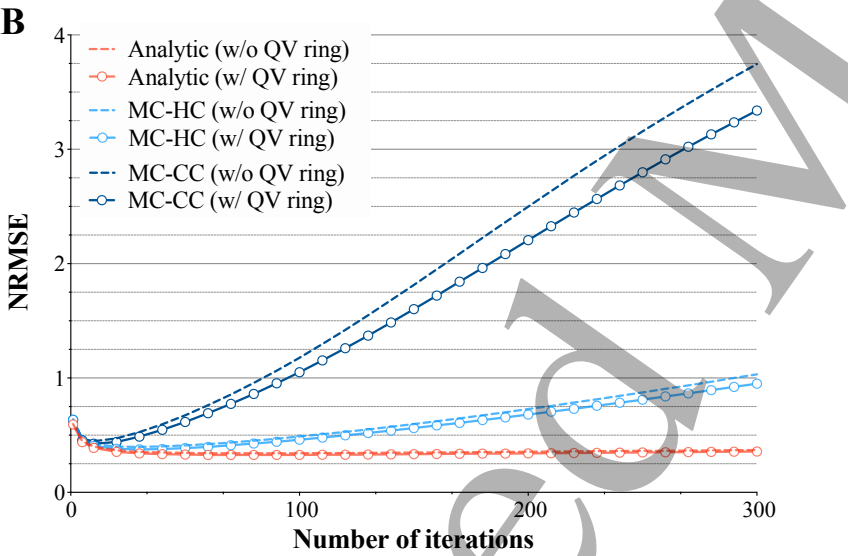

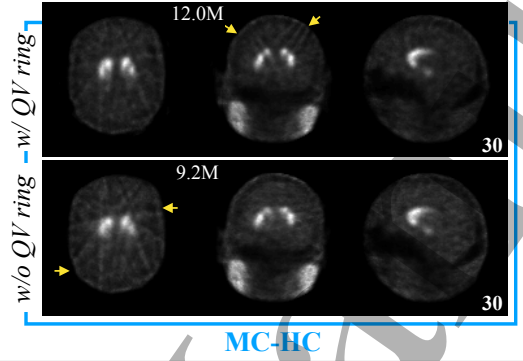

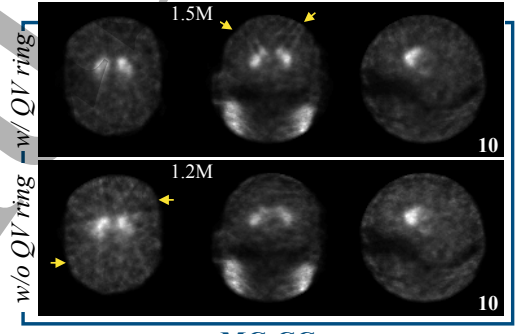

Lowest NRMSE over iterations

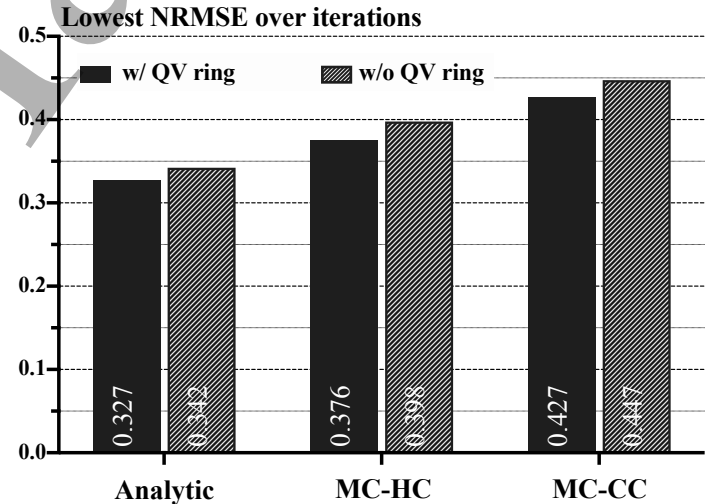

Figure 9: (A) Sample transverse, coronal, and sagittal slices of the DaTscan phantom at the iteration provided at lower right $(* *)$ showing the lowest NRMSE obtained for the noise-free analytic (left), high-count (middle) and clinical count (right) level MC scenarios with and without the QV ring. The ground truth slices are shown on the top left. For MC-CC, the reconstructed images shown are the images for a single noise realization. The yellow arrows highlight regions affected by the streak artifacts. The green arrows show the artifacts related to extra-VOI activity. The count levels ( $\mathrm{M}$ for million detected count) for each MC scenarios are shown at the upper right on the transverse slices. (B) NRMSE and lowest NRMSE values over iterations for all the investigated scenarios with and without the QV ring. Here, results are obtained from datasets consisting of counts originating from the whole-body.

\subsubsection{Evaluation of the $Q V$ ring Inclusion for DaTscan Imaging}

In Figure 9 A, we present the reconstructed images for the three scenarios (i.e., Analytic, MC-HC, and MC-CC). Similar to Sections 3.3 and 3.4, the best image quality of the brain region is obtained for the analytic noise-free scenario, for which we have a perfect match in PSF modeling despite more extra-VOI counts. However, for this scenario only, strong artifacts due to extra-VOI counts are visible at the edges of the VOI, especially at the salivary glands' locations (see green arrows on the left of Figure 9-A). Those patterns almost disappeared for realistic MC scenarios, where attenuation helps in reducing the proportion of those counts. Streak artifacts can be seen in transverse slices when the QV ring is absent, and mostly in the 
superior region of coronal slices when it is present (see yellow arrows on the middle and the right of Figure 9-A). These artifacts are related to a lack of angular sampling in relation to the intense activity of the striatal region. The addition of the QV ring improves the angular sampling and the spatial resolution, but the use of only one aperture per detector does not completely correct for these streak artifacts. With improvements in axial and angular sampling in this region by limited detector rotation (Zeraatkar et al. 2020) or the use of multiple pinholes per detector (Zeraatkar et al. 2019) these artifacts are reduced. A loss of resolution is observed in the striatal region as the statistical noise increases (i.e., compare MC-HC to MC-CC), and the number of iterations to achieve the lowest NRMSE is reduced (from 30 to 10). The QV ring addition considerably enhanced the spatial resolution and the overall image quality in the striatal region, leading to improved differentiation of the caudate from the putamen. The salivary gland regions are similarly recovered, whether the QV ring is absent or present. Despite the salivary glands being partly located within the VOI, none of the scaling artifacts in the striatal region previously mentioned in Section 3.5.1 are visible here.

In Figure 9-B, we show a plot of the NRMSE versus iteration number as well as a bar-graph of the lowest NRMSE value obtained over iterations for the three scenarios with and without the inclusion of the QV-ring. Note that the visual findings from Figure 9 A are confirmed by the NRMSE results. Quantitatively, adding the QV-ring in imaging leads to the lowest NRMSE values for each scenarios. These improvements are $4.4 \%$, $5.5 \%$, and $4.5 \%$ for the analytic, $\mathrm{MC}-\mathrm{HC}$, and $\mathrm{MC}-\mathrm{CC}$, respectively.

A
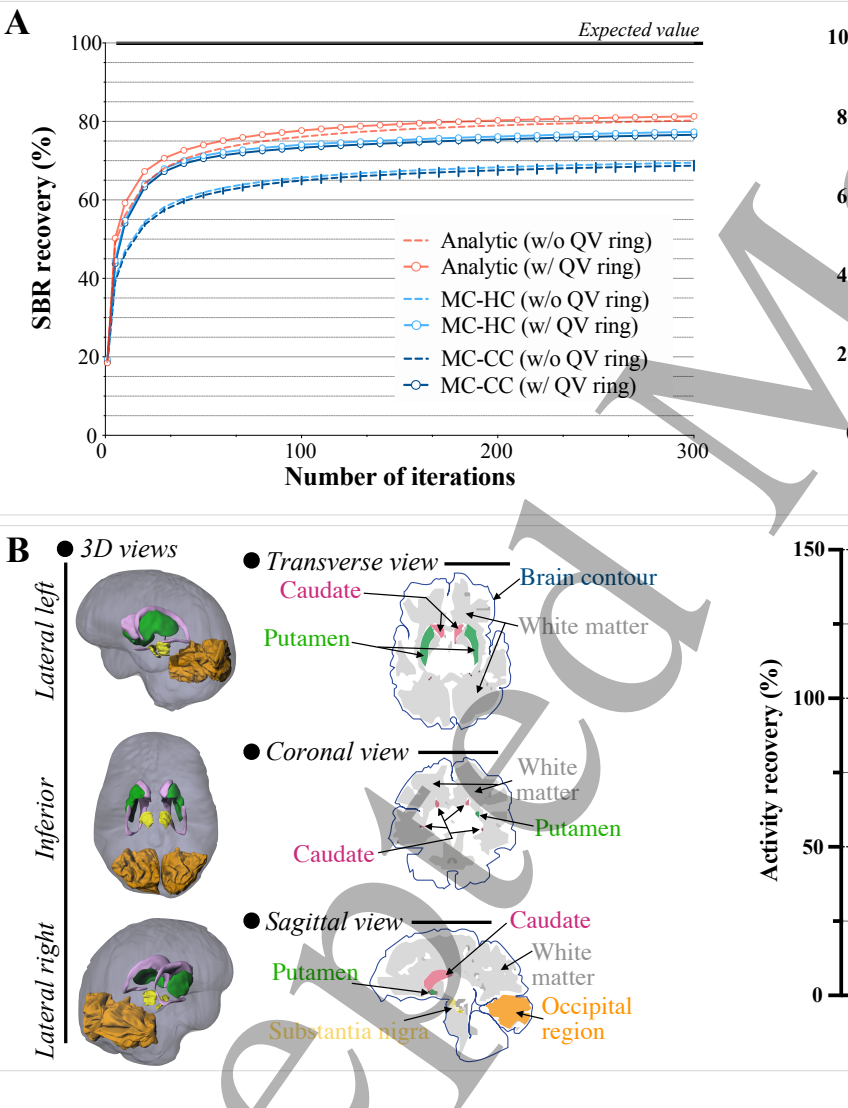

Highest SBR recovery values over iterations $(\%)$
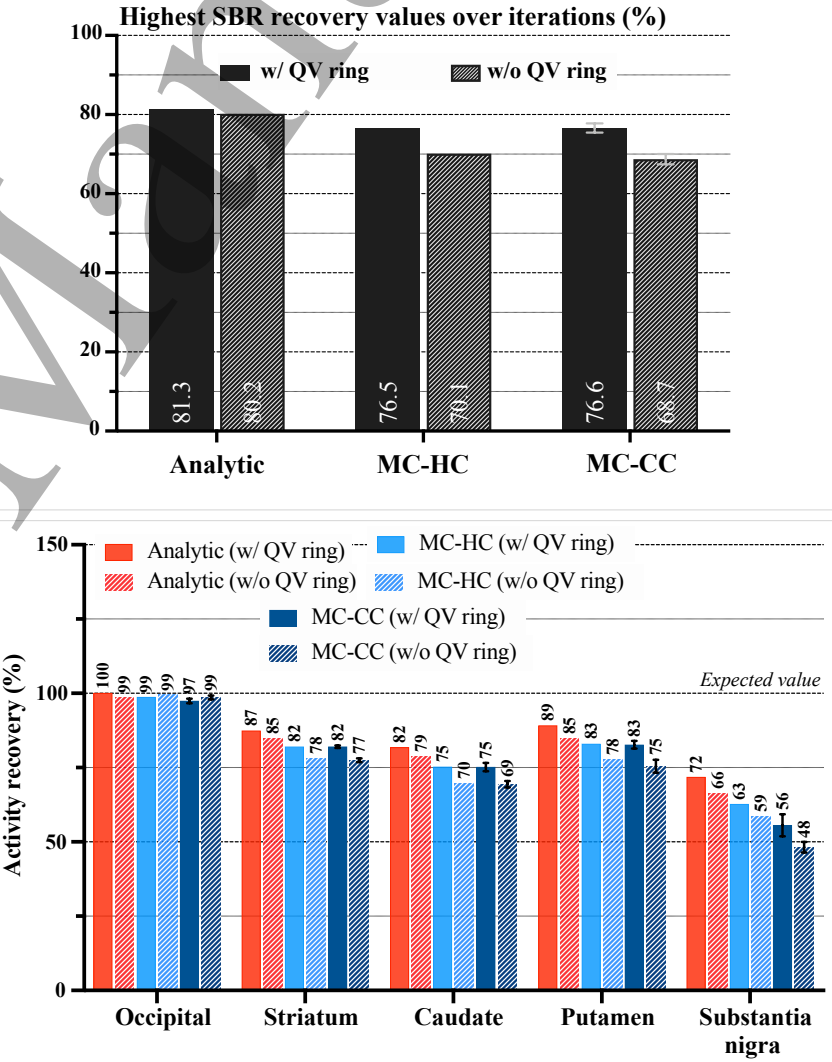

Figure 10: (A) Striatal binding ratio recovery (\%SBR) values as a function of iteration number and bar-graph comparing the highest \%SBR recovery over iteration obtained for the noise-free analytic (left), MC high-count (HC) (middle) and clinical count (CC) (right) scenarios with and without the QV ring. The datasets are based on the whole-body counts. (B) Best activity recovery (\%AR) values over iterations (i.e., closest value to $100 \%$ ) for different clinically-relevant DaTscan imaging brain regions (see anatomical definitions on the left). Note, the striatum which belongs to the grey matter is composed of the caudate, putamen and the substantia nigra. The volumes (cc) for the regions are: occipital (71.8), striatum (22), caudate (9.7), Putamen (10.2), and substantia nigra (1.3). For the MC-CC scenario, we show the average SBR, \%SBR, and \%AR values over five noise realizations and the standard deviations by bars on the plots. 
Figure 10 summarizes our quantification results. In addition to visual interpretation, quantification of the striatal uptake is highly recommended for improving diagnosis (Tossici-Bolt et al. 2006, Badiavas et al. 2011) and reducing equivocal reports (Wagner 2014). In fact, the estimation of the striatal binding ratio (SBR) is recommended by the European Association of Nuclear Medicine (EANM) Neuroimaging guideline (Darcourt et al. 2010). In addition to SBR, percent activity recovery (\% AR) of brain structures is of clinical interest (Ritt et al. 2011, Bailey \& Willowson 2013). In the following sections, we present and discuss relative improvement and degradation in SBR recovery or \%AR. The QV ring addition leads to an improvement in SBR recovery of about $1.4 \%$ for the noise-free analytic scenario (Figure 10-A). For the more realistic MC scenarios, this enhancement is higher due to the improved resolution and sampling plus the gain in sensitivity provided by the QV ring, $9.1 \%$, and $11.5 \%$ for MC-HC and MC-CC, respectively. Note that due to PVE, the \%AR values are the lowest for small structures such as substantia nigra (1.3 cc) or caudate (9.7 cc), compared to large structures such as white matter $(626.5 \mathrm{cc})$ or occipital lobe $(71.8 \mathrm{cc})$. Despite having the highest proportion of extra-VOI counts, the best \% AR values are obtained for the noise-free analytic scenario for which we considered a perfect match in PSF modeling and an absence of attenuation and ${ }^{123}$ I downscatter interaction (Figure 10-B). Structures with larger volumes, such as the occipital lobe and white matter regions, are similarly recovered with or without the QV ring. The addition of the QV ring improved \%AR values for mid and small-size brain structures, such as the striatum (by $2.4 \%$ ), caudate (by $3.8 \%$ ), putamen (by $4.7 \%$ ) and substantia nigra (by $9.1 \%$ ). Indeed, such structures with volumes below 20 cc are more affected by PVE, as demonstrated by Pacilio et al. (Pacilio et al. 2011). For the MC scenarios, due to the gain in sensitivity provided by the QV ring addition, further \% AR enhancements are achieved for the striatum (by $5.1 \%$ ), caudate (by $7.1 \%$ ), putamen (by $6.4 \%$ ), and substantia nigra (by $6.8 \%$ ). Further increases of statistical noise causing a reduction in the number of iterations (i.e., MC-CC), results in even significantly higher improvements, by $6.5 \%, 8.7 \%, 10.7 \%$, and $16.7 \%$ for the striatum, caudate, putamen, and substantia nigra respectively.

\subsubsection{Evaluation of the QV ring Inclusion for Perfusion Imaging}

In Figure 11-A, we present the reconstructed images of the perfusion distribution for the three scenarios considered (i.e., Analytic, MC-HC, and MC-CC). As was found in the DaTscan study, for the analytic scenario for which we have a perfect match in PSF modelling as well as neither statistical noise nor attenuation, artifacts are seen due to extra-VOI counts at the edges of the VOI (see yellow arrows on the left of Figure 11 A). However, these patterns are less visible than for the DaTscan for which we had a higher proportion of extra-VOI counts (see green arrows on the left of Figure 9fA). Since the analytic scheme solely modelled the ${ }^{123} \mathrm{I}-159 \mathrm{keV}$ photons imaging, this scenario is likely similar to the performance of ${ }^{99 \mathrm{~m}} \mathrm{Tc}-$ HMPAO and ${ }^{99 \mathrm{~m}} \mathrm{Tc}-\mathrm{ECD}$ imaging which have comparable bio-distributions to ${ }^{123} \mathrm{I}$-IMP. Nevertheless, given the lower principal emission energy (i.e., $140.5 \mathrm{keV}$ ), we might expect more photons originating outside of the VOI to be attenuated and scattered with subsequent detection in the photopeak window, and therefore lower impact on image quality than for ${ }^{123} \mathrm{I}$. As observed for DaTscan, these artifacts remain slightly visible for realistic MC simulations scenarios, for which attenuation considerably reduces the proportion of those counts. Nonetheless, to decrease the probability of target regions (i.e., the brain) from being impacted by such patterns located at the edges of the VOI, the patient will need to be positioned so that the top of the head is near to the superior axial extent of the VOI. Interestingly, the streak artifacts observed for the DaTscan studies are not visible on these reconstructed images, whether the QV ring is present or absent (see yellow arrows on the left of Figure 9-A). Indeed, these patterns appear to be more visible for intense structures in low-activity uniform background that are not present in perfusion. Overall, the visual image quality is considerably enhanced when the QV ring is present, especially in the striato-thalamic region. The contrast between grey and white matter regions is improved when the QV ring is included likely due to the gains in sensitivity, angular sampling, and spatial resolution. Removing the QV ring introduces considerable loss of contrast and spatial resolution in the central region of the brain, hindering the recovery of such structures.

In Figure 11 $\mathrm{B}$, we report NRMSE and lowest NRMSE values over iterations. There it can be noted that our visual findings are confirmed by the NRMSE analysis. The addition of the QV ring leads to NRMSE improvement of $4.8 \%, 3.6 \%$, and $3.3 \%$ for the analytic, $\mathrm{MC}-\mathrm{HC}$ and $\mathrm{MC}-\mathrm{CC}$ cases, respectively. Despite more extra-VOI counts, the best NRMSE values are obtained for the analytic scenario. Statistical-noise, PSFmodeling, ${ }^{123} \mathrm{I}$ downscatter interaction, and attenuation/scatter-correction inaccuracies are the predominant 


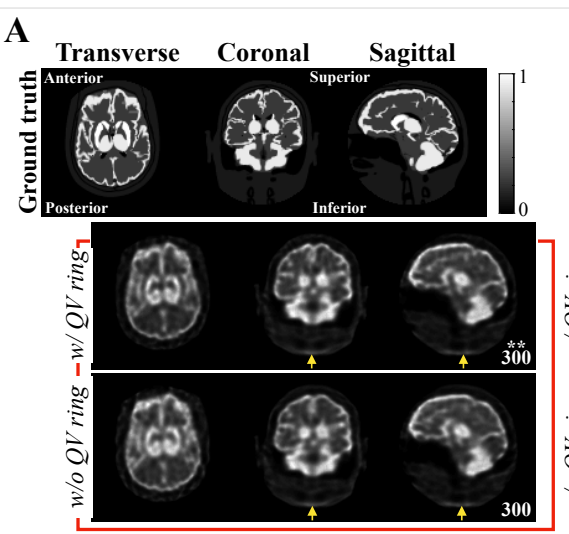

Analytic

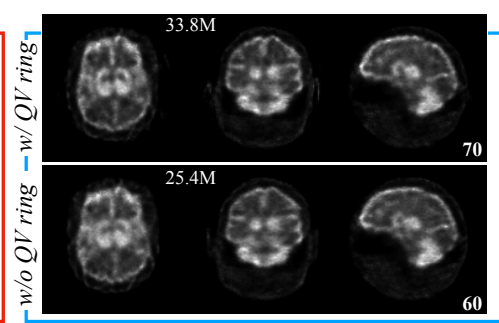

MC-HC
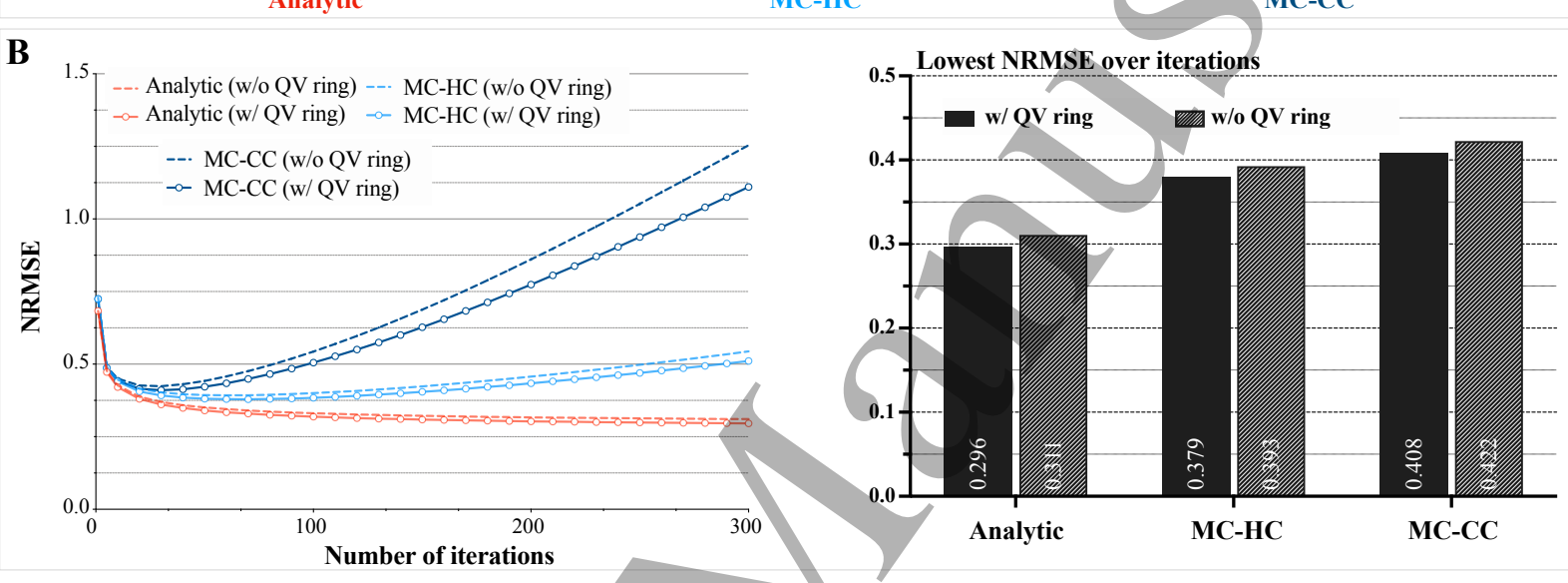

Figure 11: (A) Sample transverse, coronal, and sagittal slices of the perfusion phantom at the iteration provided at lower right $(* *)$ showing the lowest NRMSE obtained for the noise-free analytic (left), high-count (HC) (middle) and clinical count (CC) (right) level MC scenarios with and without the QV ring. The ground truth slices are shown on the top left. For MC-CC, the reconstructed images shown are the images for a single noise realization. The yellow arrows highlight the artifacts related to extra-VOI activity. The count levels (M for million detected counts) for each MC scenarios are shown at the upper right on the transverse slices. (B) NRMSE and lowest NRMSE values over iterations for all the investigated scenarios with and without the QV ring. Here, results are obtained from datasets consisting of counts originating from the whole-body.

sources of image quality degradation, compared to that attributable to extra-VOI counts.

Figure 12 summarizes our quantification results based on the activity recovery (\%AR) of brain structures relevant to perfusion imaging. Since grey matter structures present higher activity (i.e., the striatum, caudate, putamen, substantia nigra, grey matter, and cortex plus cerebellum), their \%AR were underestimated due to a spill-over of activity. This contrasts with the white matter regions for which the $\% \mathrm{AR}$ were overestimated by spill-in activity from surrounding grey matter-related structures with higher uptake. Despite the highest proportion of extra-VOI counts, the best \%AR values are obtained for the noise-free analytic scenario for which we considered a perfect PSF modelling as well as neither attenuation nor ${ }^{123} \mathrm{I}$ downscatter interaction. Large volume structures, such as the white and the grey matters or the cortex-plus-cerebellum regions are similarly recovered with or without the QV ring. The addition of the QV ring improved \%AR values for mid and small-size brain structures such as the striatum (by $3.4 \%$ ), caudate (by $3.7 \%$ ), and putamen (by 4.6\%). For MC-HC, due to the gain in sensitivity provided by the QV ring, further \%AR enhancement is achieved for the striatum (by 4.8\%), caudate (by 11.0\%), and putamen (by $6.2 \%$ ). Despite an increase in statistical noise, activity in such regions is similarly recovered for a clinical count level. For perfusion, as the clinical and high count levels were higher than that of the DaTscan, the $\% \mathrm{AR}$ values are less degraded by statistical noise. This is also seen by the smaller standard deviation values $(<5 \%$ of mean values). 

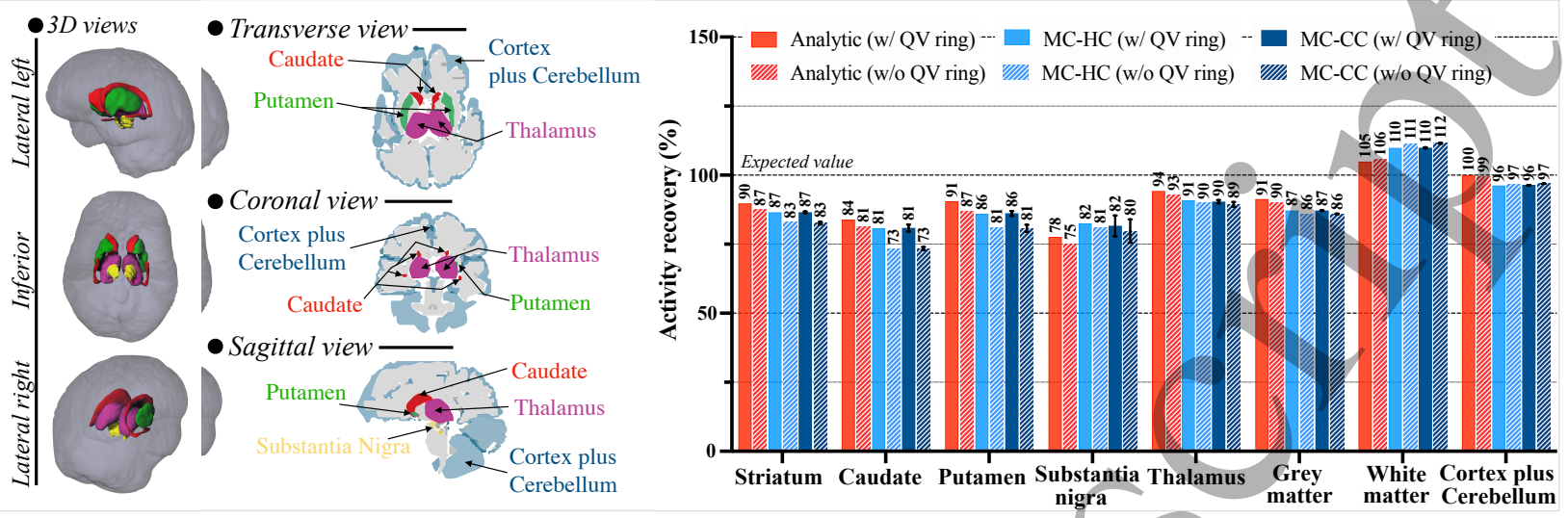

Figure 12: Best activity recovery (\%AR) values over iterations (i.e. closest value to 100\%) for selected perfusion imaging brain regions (see schema on the left). Note, the striatum which belongs to the grey matter is composed of the caudate, putamen, and the substantia nigra. Here, \%AR are obtained for datasets including extra-VOI counts. The volumes (cc) for the regions are: for striatum (22), caudate (9.7), putamen (10.2), substantia nigra (1.3), thalamus (19.12), grey matter (663.6), and white matter (626.5), cortex and cerebellum (643). Iteration numbers at which these best \%AR values are obtained were 300 for all scenarios and for all brain structures of interest. For MC-CC, the average \%AR value over five noise realizations and the standard deviation is shown by bar on the plots.

For perfusion imaging the substantia nigra experiences spill-in activity from large surrounding structures such as the thalamus, which have similar activity concentrations in the source distribution. The \%AR values are thus higher than the ones obtained for DaTscan for which thalamus had significantly lower uptake. This results in \%AR values superior for the MC scenarios than those observed for the analytic scenario. Since MC scenarios suffer from statistical noise and inaccuracies in PSF modelling, the achieved spatial resolution is worse than that of the noise-free analytic scenario leading to an increased spill-in activity.

\section{Conclusions}

Understandably, there has been skepticism expressed by some in the brain SPECT imaging research community regarding the utility of the vertex and "quasi" vertex views. Given their location, without attenuation or scatter, one would expect more detection of photons originating from areas outside the VOI and thereby degradation in image quality. However, as demonstrated herein through analytic and MonteCarlo simulations of ${ }^{123} \mathrm{I}$ source distributions, the contribution of extra-VOI activity is greatly diminished due to attenuation and scatter in the patient's body. Thus, we have shown that the addition of quasi-vertex views considerably improves the image quality visually and in terms of clinically relevant quantitative metrics, such as the striatal binding ratio, and the recovery of activity in selected brain structures. Furthermore, system sensitivity as well as angular sampling, spatial resolution, and uniformity in the brain region are enhanced by the presence of such additional detectors.

\section{Acknowledgment}

A preliminary report of ideas and initial results in this manuscript was presented at the 2017 IEEE Nuclear Science Symposium and Medical Imaging Conference and published in the conference proceeding by J.C. Goding, B. Auer et al. as "An Investigation of Quasi-Vertex Views in Brain SPECT Imaging-Initial Results". Research reported herein was supported by the National Institute of Biomedical Imaging and Bioengineering of the National Institutes of Health under Award Number R01 EB022521. The content is the sole responsibility of the authors and does not necessarily represent the official views of the National Institutes of Health.

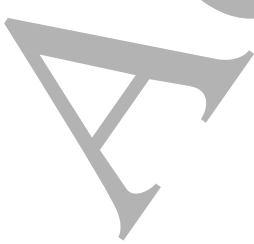




\section{References}

Accorsi, R. \& Metzler, S. D. (2004). Analytic determination of the resolution-equivalent effective diameter of a pinhole collimator, IEEE transactions on medical imaging 23(6): 750-763.

Adriaanse, S. M., de Wit, T. C., Stam, M., Verwer, E., de Bruin, K. M. \& Booij, J. (2018). Clinical evaluation of [ ${ }^{123}$ I] FP-CIT SPECT scans on the novel brain-dedicated InSPira HD SPECT system: a head-to-head comparison, EJNMMI research 8(1): 1-6.

Amen, D. G., Highum, D., Licata, R., Annibali, J. A., Somner, L., Pigott, H. E., Taylor, D. V., Trujillo, M., Newberg, A., Henderson, T. \& Willeumier, K. (2012). Specific ways brain SPECT imaging enhances clinical psychiatric practice, J. Psychoactive Drugs 44(2): 96-106.

Auer, B., De Beenhouwer, J., Kalluri, K., Goding, J. C., Furenlid, L. R. \& King, M. A. (2018). Preliminary investigation of design parameters of an innovative multi-pinhole system dedicated to brain spect imaging, 2018 IEEE Nuclear Science Symposium and Medical Imaging Conference Proceedings (NSS/MIC), IEEE, pp. 1-3.

Auer, B., Könik, A., Kalluri, K., De Beenhouwer, J., Furenlid, L. R. \& King, M. A. (2018). Preliminary evaluation of surface mesh modeling of system geometry, anatomy phantom, and source activity for gate simulations, 2018 IEEE Nuclear Science Symposium and Medical Imaging Conference Proceedings (NSS/MIC), IEEE, pp. 1-3.

Auer, B., Zeraatkar, N., De Beenhouwer, J., Kalluri, K., Kuo, P. H., Furenlid, L. R. \& King, M. A. (2019). Investigation of a Monte-Carlo simulation and an analytic-based approach for modeling the system response for clinical I-123 brain SPECT imaging, 15th International Meeting on Fully Three-Dimensional Image Reconstruction in Radiology and Nuclear Medicine, Vol. 11072, International Society for Optics and Photonics, p. 1107214.

Badiavas, K., Molyvda, E., Iakovou, I., Tsolaki, M., Psarrakos, K. \& Karatzas, N. (2011). SPECT imaging evaluation in movement disorders: Far beyond visual assessment, Eur. J. Nucl. Med. Mol. Imaging 38(4): 764-773.

Bailey, D. L. \& Willowson, K. P. (2013). An evidence-based review of quantitative SPECT imaging and potential clinical applications, J. Nucl. Med. 54(1): 83-89.

Bajaj, N., Hauser, R. A. \& Grachev, I. D. (2013). Clinical utility of dopamine transporter single photon emission CT (DaTSPECT) with $\left({ }^{123} \mathrm{I}\right)$ ioflupane in diagnosis of parkinsonian syndromes, J. Neurol. Neurosurg. Psychiatry 84(11): 12881295.

Banks, K. P., Peacock, J. G., Clemenshaw, M. N. \& Kuo, P. H. (2019). Optimizing the Diagnosis of Parkinsonian Syndromes With ${ }^{123}$ I-Ioflupane Brain SPECT, Am. J. Roentgenol. 213(2): 243-253.

Barrett, H. H., Furenlid, L. R., Freed, M., Hesterman, J. Y., Kupinski, M. A., Clarkson, E. \& Whitaker, M. K. (2008). Adaptive SPECT, IEEE Trans. Med. Imaging 27(6): 775-788.

Batis, J. (2011). Performance characteristics of the inSPira HD, a new generation brain-dedicated SPECT camera, J. Nucl. Med. 52(1): 1934.

Beekman, F. J., Van Der Have, F., Goorden, M. C., Vaissier, P. E., Van Roosmalen, J., During, H. \& Vastenhouw, B. (2015). G-SPECT-I: a full ring high sensitivity and ultra-fast clinical molecular imaging system with $<3 \mathrm{~mm}$ resolution, Eur. J. Nucl. Med. Mol. Imaging 42: 209.

Beekman, F. \& van der Have, F. (2007). The pinhole: gateway to ultra-high-resolution three-dimensional radionuclide imaging, Eur. J. Nucl. Med. Mol. Imaging 34(2): 151-161.

Bonne, O., Krausz, Y. \& Lerer, B. (1992). SPECT imaging in psychiatry. A review, Gen. Hosp. Psychiatry 14(5): 296-306.

Camargo, E. E. (2001). Brain SPECT in neurology and psychiatry, J. Nucl. Med. 42(4): 611-623.

Catafau, A. M. (2001). Brain SPECT in clinical practice. Part I: Perfusion, J. Nucl. Med. 42(2): 259-271.

Chen, L., Tsui, B. M. W. \& Mok, G. S. P. (2017). Design and evaluation of two multi-pinhole collimators for brain SPECT, Ann. Nucl. Med. 31(8): 636-648.

Chen, Y., Goorden, M. C., Vastenhouw, B. \& Beekman, F. J. (2019). Optimized Sampling for High Resolution Multi-Pinhole Brain SPECT with Stationary Detectors, Phys. Med. Biol.

Chen, Y., Vastenhouw, B., Wu, C., Goorden, M. C. \& Beekman, F. J. (2018). Optimized image acquisition for dopamine transporter imaging with ultra-high resolution clinical pinhole SPECT, Phys. Med. Biol. 63(22).

Darcourt, J., Booij, J., Tatsch, K., Varrone, A., Vander Borght, T., Kapucu, Ö. L., Någren, K., Nobili, F., Walker, Z. \& Van Laere, K. (2010). EANM procedure guidelines for brain neurotransmission SPECT using ${ }^{123}$ I-labelled dopamine transporter ligands, version 2, Eur. J. Nucl. Med. Mol. Imaging 37(2): 443-450.

Djang, D. S., Janssen, M.J., Bohnen, N., Booij, J., Henderson, T. A., Herholz, K., Minoshima, S., Rowe, C. C., Sabri, O., Seibyl, J., Van Berckel, B. N. \& Wanner, M. (2012). SNM practice guideline for dopamine transporter imaging with ${ }^{123}$ I-ioflupane SPECT 1.0, J. Nucl. Med. 53(1): 154-163.

El Fakhri, G., Ouyang, J., Zimmerman, R. E., Fischman, A. J. \& Kijewski, M. F. (2006). Performance of a novel collimator for high-sensitivity brain SPECT, Medical physics 33(1): 209-215.

Erlandsson, K., Buvat, I., Pretorius, P. H., Thomas, B. A. \& Hutton, B. F. (2012). A review of partial volume correction techniques for emission tomography and their applications in neurology, cardiology and oncology, Phys. Med. Biol. 57(21): R119.

Farid, K., Caillat-Vigneron, N. \& Sibon, I. (2011). Is brain SPECT useful in degenerative dementia diagnosis?, J. Comput. Assist. Tomogr. 35(1): 1-3.

Farncombe, T. H., Gifford, H. C., Narayanan, M. V., Pretorius, P. H., Frey, E. C. \& King, M. A. (2004). Assessment of scatter compensation strategies for ${ }^{67} \mathrm{Ga}$ SPECT using numerical observers and human LROC studies, Journal of Nuclear Medicine 45(5): 802-812.

Fukuma, K., Kajimoto, K., Tanaka, T., Takaya, S., Kobayashi, K., Shimotake, A., Matsumoto, R., Ikeda, A., Toyoda, K. \& Ihara, M. (2020). Visualizing prolonged hyperperfusion in post-stroke epilepsy using postictal subtraction SPECT, $J$. Cereb. Blood Flow Metab. (February).

Gonzalez, A. J., Sanchez, F. \& Benlloch, J. M. (2018). Organ-Dedicated Molecular Imaging Systems, IEEE Trans. Radiat. Plasma Med. Sci. 2(5): 388-403. 
Goorden, M. C., Rentmeester, M. C. \& Beekman, F. J. (2009). Theoretical analysis of full-ring multi-pinhole brain SPECT, Phys. Med. Biol. 54(21): 6593-6610.

Gullberg, G. T., Reutter, B. W., Sitek, A., Maltz, J. S. \& Budinger, T. F. (2010). Dynamic single photon emission computed tomography-basic principles and cardiac applications, Phys. Med. Biol. 55(20).

Holmes, R. A. (1971). Value of the vertex view in brain scanning, Semin. Nucl. Med., Vol. 1, Elsevier, pp. 48-55.

Holmes, R. A. \& North, W. A. (1969). The vertex view in routine brain scanning, Am. J. Roentgenol. 106(2): $347-353$.

Ito, H., Inoue, K., Goto, R., Kinomura, S., Taki, Y., Okada, K., Sato, K., Sato, T., Kanno, I. \& Fukuda, H. (2006). Database of normal human cerebral blood flow measured by SPECT: I. Comparison between I-123-IMP, Tc-99m-HMPAO, and Tc-99m-ECD as referred with O-15 labeled water PET and voxel-based morphometry, Ann. Nucl. Med. 20(2): $131-138$.

Ito, H., Sato, T., Odagiri, H., Inoue, K., Shidahara, M., Suhara, T., Hatazawa, J. \& Fukuda, H. (2006). Brain and whole body distribution of N-isopropyl-4-iodoamphetamine (I-123) in humans: Comparison of radiopharmaceuticals marketed by different companies in Japan, Ann. Nucl. Med. 20(7): 493-498.

Jan, S., Santin, G., Strul, D., Staelens, S., Assie, K., Autret, D., Avner, S., Barbier, R., Bardies, M. \& Bloomfield, P. M. (2004). GATE: a simulation toolkit for PET and SPECT, Phys. Med. Biol. 49(19): 4543.

Juni, J. E., Waxman, A. D., Devous, M. D., Tikofsky, R. S., Ichise, M., Van Heertum, R. L., Carretta, R. F. \& Chen, C. C. (2009). Procedure guideline for brain perfusion SPECT using ${ }^{99 m}$ Tc radiopharmaceuticals 3.0, J. Nucl. Med. Technol. 37(3): 191-195.

Kant, R., Smith-Seemiller, L., Isaac, G. \& Duffy, J. (1997). Tc-HMPAO SPECT in persistent post-concussion syndrome after mild head injury comparison with MRI/CT, Brain Inj. 11(2): 115-124.

Kessler, R. M., Ellis, J. J. \& Eden, M. (1984). Analysis of emission tomographic scan data: limitations imposed by resolution and background., Journal of computer assisted tomography 8(3): 514-522.

Khalil, M. M., Tremoleda, J. L., Bayomy, T. B. \& Gsell, W. (2011). Molecular SPECT Imaging: An Overview, Int. J. Mol. Imaging 2011: 1-15.

King, M. A., Mukherjee, J. M., Könik, A., Zubal, I. G., Dey, J. \& Licho, R. (2016). Design of a Multi-Pinhole Collimator for I-123 DaTscan Imaging on Dual-Headed SPECT Systems in Combination with a Fan-Beam Collimator, IEEE Trans. Nucl. Sci. 63(1): 90-97.

Kogure, D., Matsuda, H., Ohnishi, T., Asada, T., Uno, M., Kunihiro, T., Nakano, S. \& Takasaki, M. (2000). Longitudinal evaluation of early Alzheimer's disease using brain perfusion SPECT, J.Nucl. Med. 41(7): 1155-1162.

Könik, A., Auer, B., De Beenhouwer, J., Kalluri, K., Zeraatkar, N., Furenlid, L. R. \& King, M. A. (2019). Primary, scatter, and penetration characterizations of parallel-hole and pinhole collimators for I-123 SPECT, Phys. Med. Biol. 64(24).

Könik, A., Beenhouwer, J. D., Mukherjee, J. M., Kalluri, K., Banerjee, S., Zeraatkar, N., Fromme, T. \& King, M. A. (2018). Simulations of a Multipinhole SPECT Collimator for Clinical Dopamine Transporter (DAT) Imaging, IEEE Trans. Radiat. Plasma Med. Sci. 2(5): 444-451.

Könik, A., Zeraatkar, N., Kalluri, K., Auer, B., Furenlid, L. R. \& King, M. A. (2019). Improved Quantitative DAT Imaging with Multiplexing in a Multi-pinhole SPECT System, J. Nucl. Med. 60(1): 524.

Kuo, P. H., Lei, H. H., Avery, R., Krupinski, E. A., Bauer, A., Sherman, S., McMillan, N., Seibyl, J. \& Zubal, G. I. (2014). Evaluation of an objective striatal analysis program for determining laterality in uptake of ${ }^{123}$ I-ioflupane SPECT images: comparison to clinical symptoms and to visual reads, J. Nucl. Med. Technol. 42(2): 105-108.

Kuribara, T., Enatsu, R., Kitagawa, M., Arihara, M., Sasagawa, A., Hirano, T., Suzuki, H., Yokoyama, R., Mikami, T. \& Mikuni, N. (2020). Neuroimaging and neurophysiological evaluation of severity of Parkinson's disease, J. Clin. Neurosci. .

Lederer, C. M. \& Shirley, V. S. (1978). Table of Isotopes, 7th edn Wiley, New York .

Lee, T. C., Ellin, J. R., Huang, Q., Shrestha, U., Gullberg, G. T. \& Seo, Y. (2014). Multipinhole collimator with 20 apertures for a brain SPECT application, Med. Phys. 41(11): 1-7.

Li, N. \& Meng, L. J. (2011). Adaptive angular sampling for SPECT imaging, IEEE Trans. Nucl. Sci. 58(5 PART 1): $2205-2218$.

Li, X., Zubal, G., King, M. A. \& Furenlid, L. R. (2017). Design of a modular spect camera with improved spatial resolution near edges, 2017 IEEE Nuclear Science Symposium and Medical Imaging Conference (NSS/MIC), IEEE, pp. 1-4.

Matsuda, H., Mizumura, S., Nagao, T., Ota, T., Iizuka, T., Nemoto, K., Takemura, N., Arai, H. \& Homma, A. (2007). Automated discrimination between very early Alzheimer disease and controls using an easy Z-score imaging system for multicenter brain perfusion single-photon emission tomography, Am. J. Neuroradiol. 28(4): 731-736.

Montoya, A., Price, B. H., Menear, M. \& Lepage, M. (2006). Brain imaging and cognitive dysfunctions in Huntington's disease, J. Psychiatry Neurosci. 31(1): 21-29.

Moore, S. C., Kouris, K. \& Cullum, I. (1992). Collimator design for single photon emission tomography, European journal of nuclear medicine 19(2): 138-150.

Moretti, J. L., Tamgac, F., Weinmann, P., Caillat-Vigneron, N., Belin, C. A., Cesaro, P., Holman, B. L. \& Defer, G. (1994). Early and delayed brain SPECT with technetium-99m-ECD and iodine-123-IMP in subacute strokes, J. Nucl. Med. 35(9): 1444-1449.

Ng, C. Y., Clinthorne, N. H., Fessler, J. A., Hero, A. O., Zhang, Y. \& Rogers, W. L. (1996). Preliminary studies on the feasibility of addition of vertex view to conventional brain SPECT, 1996 IEEE Nucl. Sci. Symp. Conf. Rec., Vol. 2, IEEE, pp. 1057-1061.

Nillius, P. \& Danielsson, M. (2010). Theoretical bounds and system design for multipinhole SPECT, IEEE Trans. Med. Imaging 29(7): $1390-1400$.

Nyquist, H. (1928). Certain topics in telegraph transmission theory, Trans. Am. Inst. Electr. Eng. 47(2): 617-644.

Ogawa, K. \& Ichimura, Y. (2014). Simulation study on a stationary data acquisition SPECT system with multi-pinhole collimators attached to a triple-head gamma camera system, Ann. Nucl. Med. 28(8): 716-724.

Overton, M. C., Haynie, T. P., Otte, W. K. \& Coe, J. E. (1965). The vertex view in brain scanning, Journal of Nuclear Medicine 6(10): 705-710.

Pacilio, M., Basile, C., Shcherbinin, S., Caselli, F., Ventroni, G., Aragno, D., Mango, L. \& Santini, E. (2011). An innovative iterative thresholding algorithm for tumour segmentation and volumetric quantification on SPECT images: Monte 
Carlo-based methodology and validation, Med. Phys. 38(6Part1): 3050-3061.

Patil, S. \& Ravi, B. (2005). Voxel-based representation, display and thickness analysis of intricate shapes, Ninth Int. Conf. Comput. Aided Des. Comput. Graph., IEEE, pp. 6-pp.

Petrella, J. R., Coleman, R. E. \& Doraiswamy, P. M. (2003). State of the Art Radiology Neuroimaging and Early Diagnosis of Alzheimer Disease : A Look to the Future 1, Radiology 226(2): 315-336.

Pimlott, S. L. \& Sutherland, A. (2011). Molecular tracers for the PET and SPECT imaging of disease, Chem. Soc. Rev. 40(1): 149-162.

Poston, A. (2000). Human engineering design data digest, Washington, DC Dep. Def. Hum. Factors Eng. Tech. Advis. Gr. pp. $61-75$.

Rault, E., Staelens, S., Van Holen, R., De Beenhouwer, J. \& Vandenberghe, S. (2010). Accurate Monte Carlo modelling of the back compartments of SPECT cameras, Phys. Med. Biol. 56(1): 87.

Ritt, P., Vija, H., Hornegger, J. \& Kuwert, T. (2011). Absolute quantification in SPECT, Eur. J. Nucl. Med. Mol. Imaging 38(SUPPL. 1): 69-77.

Rojas, A., Górriz, J. M., Ramírez, J., Illán, I. A., Martínez-Murcia, F. J., Ortiz, A., Río, M. G. \& Moreno-Caballero, M. (2013). Application of empirical mode decomposition (EMD) on DaTSCAN SPECT images to explore Parkinson disease, Expert Syst. Appl. 40(7): 2756-2766.

Santra, A. \& Kumar, R. (2014). Brain perfusion single photon emission computed tomography in major psychiatric disorders: From basics to clinical practice, Indian J. Nucl. Med. 29(4): 210-221.

Segars, W. P., Sturgeon, G., Mendonca, S., Grimes, J. \& Tsui, B. M. W. (2010). 4D XCAT phantom for multimodality imaging research, Med. Phys. 37(9): 4902-4915.

Shannon, C. E. (1949). A theorem on coloring the lines of a network, J. Math. Phys. 28(1-4): 148-152.

Shepp, L. A. \& Vardi, Y. (1982). Maximum likelihood reconstruction for emission tomography, IEEE Trans. Med. Imaging 1(2): 113-122.

Stam, M. K., Verwer, E. E., Booij, J., Adriaanse, S. M., de Bruin, C. M. \& de Wit, T. C. (2018). Performance evaluation of a novel brain-dedicated SPECT system, EJNMMI Phys. 5(1): 1-14.

Stoddart, H. A. \& Stoddart, H. F. (1992). New multidimensional reconstructions for the 12-detector, scanned focal point, single-photon tomograph, Phys. Med. Biol. 37(3): 579-586.

Suwijn, S. R., van Boheemen, C. J., de Haan, R. J., Tissingh, G., Booij, J. \& de Bie, R. M. (2015). The diagnostic accuracy of dopamine transporter SPECT imaging to detect nigrostriatal cell loss in patients with Parkinson's disease or clinically uncertain parkinsonism: A systematic review, EJNMMI Res. 5(1): 1-8.

Sydoff, M., Lizana, H., Mattsson, S., Stabin, M. G. \& Leide-Svegborn, S. (2013). Determination of the biodistribution and dosimetry of 123I-FP-CIT in male patients with suspected Parkinsonism or Lewy body dementia using planar and combined planar and SPECT/CT imaging, Appl. Radiat. Isot. 82: 300-307.

Tossici-Bolt, L., Hoffmann, S. M., Kemp, P. M., Mehta, R. L. \& Fleming, J. S. (2006). Quantification of [123I]FP-CIT SPECT brain images: An accurate technique for measurement of the specific binding ratio, Eur. J. Nucl. Med. Mol. Imaging 33(12): 1491-1499.

Van Audenhaege, K., Van Holen, R., Vandenberghe, S., Vanhove, C., Metzler, S. D. \& Moore, S. C. (2015). Review of SPECT collimator selection, optimization, and fabrication for clinical and preclinical imaging, Med. Phys. 42(8): 4796-4813.

Van Audenhaege, K., Vandenberghe, S., Deprez, K., Vandeghinste, B. \& Van Holen, R. (2013). Design and simulation of a full-ring multi-lofthole collimator for brain SPECT, Phys. Med. Biol. 58(18): 6317-6336.

Van Paesschen, W., Dupont, P., Sunaert, S., Goffin, K. \& Van Laere, K. (2007). The use of SPECT and PET in routine clinical practice in epilepsy, Curr. Opin. Neurol. 20(2): 194-202.

Wagner, D. R. P. A. U. P. H. A. U. R. C. A. U. S. N. A. U. A.-M. Q. A. U. T. W. A. U. T. (2014). Automated quantification with BRASS reduces equivocal reporting of DaTSCAN (123I-FP-CIT) SPECT studies.

Warwick, J. M. (2004). Imaging of brain function using SPECT, Metab. Brain Dis. 19(1-2): 113-123.

Yeo, J. M., Lim, X., Khan, Z. \& Pal, S. (2013). Systematic review of the diagnostic utility of SPECT imaging in dementia, Eur. Arch. Psychiatry Clin. Neurosci. 263(7): 539-552.

Zanzonico, P. (2008). Routine quality control of clinical nuclear medicine instrumentation: A brief review, J. Nucl. Med. 49(7): 1114-1131.

Zeng, G. L. (2011). Gibbs artifact reduction by nonnegativity constraint, J. Nucl. Med. Technol. 39(3): 213-219.

Zeraatkar, N., Auer, B., Kalluri, K., Momsen, N., Furenlid, L., Kuo, P. \& King, M. (2019). Effect of truncated overlapping projection data in a multi-pinhole brain spect system with temporal shuttering of apertures, Journal of Nuclear Medicine 60(supplement 1): 39-39.

Zeraatkar, N., Farahani, M. H., Rahmim, A., Sarkar, S. \& Ay, M. R. (2016). Design and assessment of a novel SPECT system for desktop open-gantry imaging of small animals: A simulation study, Med. Phys. 43(5): 2581-2597.

Zeraatkar, N., Kalluri, K. S., Auer, B., Könik, A., Fromme, T. J., Furenlid, L. R., Kuo, P. H. \& King, M. A. (2020). Investigation of Axial and Angular Sampling in Multi-Detector Pinhole-SPECT Brain Imaging, IEEE Transactions on Medical Imaging .

Zhao, H. \& Reader, A. J. (2003). Fast ray-tracing technique to calculate line integral paths in voxel arrays, 2003 IEEE Nucl. Sci. Symp. Conf. Rec. (IEEE Cat. No. 03CH37515), Vol. 4, IEEE, pp. 2808-2812. 